\title{
Recent Advances in Integrated Electrode for Electrocatalytic Carbon Dioxide Reduction
}

\author{
Yuke Song, Wenfu Xie *, Mingfei Shao * \\ State Key Laboratory of Chemical Resource Engineering, College of Chemistry, Beijing University of Chemical Technology, \\ Beijing 100029, China.
}

\begin{abstract}
The electrocatalytic carbon dioxide reduction reaction $\left(\mathrm{E}-\mathrm{CO}_{2} \mathrm{RR}\right)$ has attracted attention in recent years for its ability to effectively alleviate the environmental problems caused by the rapid increase of $\mathrm{CO}_{2}$ in the atmosphere and transform $\mathrm{CO}_{2}$ into high value-added fuels or chemicals (e.g., $\mathrm{CO}, \mathrm{HCOOH}, \mathrm{CH}_{4}, \mathrm{CH}_{3} \mathrm{OH}, \mathrm{C}_{2} \mathrm{H}_{4}, \mathrm{C}_{2} \mathrm{H}_{5} \mathrm{OH}$, etc.) under mild conditions. In addition, clean energy sources, such as solar and wind energy, can provide electrical energy for the electrochemical $\mathrm{CO}_{2}$ conversion technology used in large-scale industrial applications. One limitation of the $\mathrm{E}-\mathrm{CO}_{2} \mathrm{RR}$ is that $\mathrm{CO}_{2}$ is a thermodynamically stable linear molecule with a slow kinetic reaction rate. In addition, the $\mathrm{E}-\mathrm{CO}_{2} \mathrm{RR}$ involves complex processes, such as gas diffusion and multi-electron transfer, making its selectivity problematic. Therefore, constructing highly efficient and stable catalytic electrodes has become a core

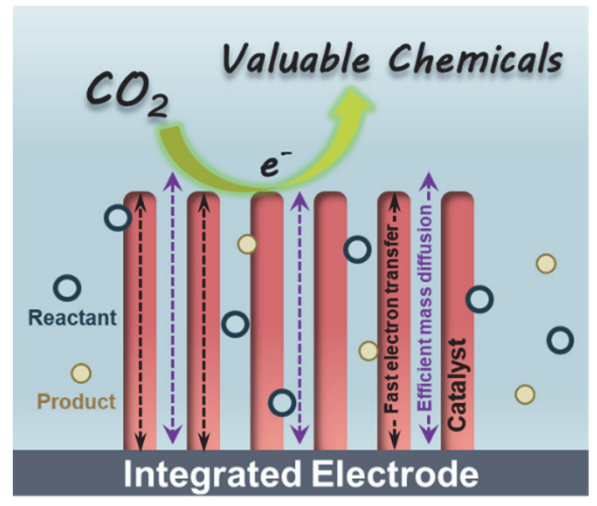
research topic in the field of $\mathrm{E}-\mathrm{CO}_{2} \mathrm{RR}$. Unfortunately, the traditional method of coating electrodes with binders (e.g., Nafion, polyvinylidene fluoride, and polytetrafluoroethylene) usually results in a low utilization ratio of active sites due to the easy aggregation of the catalysts themselves. This could result in the severe embedding of active sites and limited mass transfer. Moreover, the dissolution of the catalyst layer during the electrocatalytic process also reduces the activity and stability of the electrodes, making it difficult to reuse. Therefore, it is necessary to regulate the electrode reaction interface to improve the utilization ratio of active sites. The integrated electrodes, where the catalyst is grown directly on the current collector, can avoid the use of binders to facilitate the exposure of active sites and transfer of electrons. The integrated structure can also enhance the bonding strength between the active material and current collector and improve the cycling stability of the electrodes. Meanwhile, the micro-environment (e.g., $\mathrm{pH}$, concentration of $\mathrm{CO}_{2}$, and intermediates) at the three-phase interface can be effectively controlled on the integrated electrodes, which can enhance the performance of the $\mathrm{E}-\mathrm{CO} \mathrm{RR}_{2}$. In recent years, encouraging progress has been achieved in the study of the $\mathrm{E}-\mathrm{CO}_{2} \mathrm{RR}$. However, current reviews of the $\mathrm{E}-\mathrm{CO}_{2} \mathrm{RR}$ mainly focus on the regulation of the intrinsic activity of catalysts; discussions and reviews from the perspective of the electrodes are rarely reported. This article reviews the latest research of the integrated electrodes for the $\mathrm{E}-\mathrm{CO} \mathrm{O}_{2} \mathrm{RR}$ with a focus on the application of different types of integrated electrodes (e.g., metal, alloy, metal oxide, metal sulfide/phosphide, and metal single atom). It also analyzes the effects of morphology, surface, and interface regulation on the electrocatalytic performance of the $\mathrm{E}-\mathrm{CO}_{2} \mathrm{RR}$. Finally, it highlights the challenges that still exist in this field and discusses the future development of the integrated electrodes.
\end{abstract}

Key Words: Integrated electrode; Electrocatalysis; Carbon dioxide reduction reaction; Structural design; Interface regulation

\footnotetext{
Received: January 15, 2021; Revised: February 15, 2021; Accepted: February 22, 2021; Published online: March 1, 2021.

*Corresponding authors. Emails: wenfu2010qd@126.com (W.X.); shaomf@mail.buct.edu.cn (M.S.).

The project was supported by the National Natural Science Foundation of China (21922501, 22090031, 21871021), the Beijing Natural Science Foundation (2192040), and the Fundamental Research Funds for the Central Universities (XK1802-6, XK1803-05).

国家自然科学基金(21922501, 22090031, 21871021), 北京市自然科学基金(2192040)和中央高校基本科研业务费专项资金(XK1802-6, XK1803-05)资助 项目

(C) Editorial office of Acta Physico-Chimica Sinica
} 


\title{
一体化电极电催化二氧化碳还原研究进展
}

\author{
宋雨珂, 谢文富 ${ }^{*}$, 邵明飞* \\ 北京化工大学化学学院，化工资源有效利用国家重点实验室，北京 100029
}

\begin{abstract}
摘要: 电催化二氧化碳还原反应 $\left(\mathrm{E}-\mathrm{CO}_{2} \mathrm{RR}\right)$ 可在温和条件下将 $\mathrm{CO}_{2}$ 转化成高附加值燃料或化学品, 近年来受到广泛关注, 其在实际反应中涉及到气体扩散和多电子转移等复杂过程, 构筑高效、稳定的催化电极是其发展的核心之一。然而, 传 统涂敷电极制备时, 需要将催化剂与粘结剂混合涂覆于集流体表面, 此过程会造成活性位点包埋和传质过程受限, 致使 催化剂活性位利用率下降, 同时在反应过程中电极表面容易粉化, 造成稳定性下降, 难以重复利用。因此, 如何调控电 极反应界面, 提升催化剂活性位的利用率仍面临挑战。将催化剂原位生长于集流体上得到的一体化电极可直接应用于电 催化反应, 不仅有利于提升活性位利用率以及电荷传输能力, 还能有效调控三相界面处的微观反应环境(如 $\mathrm{pH}$ 、反应物 及反应中间体的浓度等), 从而实现电催化性能强化。本文综述了一体化电极用于 $\mathrm{E}-\mathrm{CO}_{2} \mathrm{RR}$ 的最新进展, 分析了结构和 表界面调控对 $E-\mathrm{CO}_{2} R R$ 性能的影响规律, 并对该领域仍然存在的挑战和未来一体化E- $\mathrm{CO}_{2} R R$ 电极的发展进行了评述与 展望。
\end{abstract}

关键词: 一体化电极; 电催化; 二氧化碳还原反应; 结构化设计; 表界面调控 中图分类号: 0646

\section{1 引言}

工业革命以来, 化石燃料(煤、石油、天然气 等)的过度使用造成大气中的 $\mathrm{CO}_{2}$ 含量逐年上升, 已 严重超过自然界的碳循环限度, 引起了一系列环 境问题, 如气温升高和气候剧变 ${ }^{1-4}$ 。 $\mathrm{CO}_{2}$ 转化技术, 不仅可以有效缓解 $\mathrm{CO}_{2}$ 剧增所带来的环境问题, 还 可以制备高附加值的燃料或化学品 5,6。迄今为止, $\mathrm{CO}_{2}$ 转化技术可划分为四类: 生物化学法、热化学 法、光化学法和电化学法 ${ }^{7-12}$ 。其中, 电化学 $\mathrm{CO}_{2}$ 转 化技术凭借反应条件相对温和、转化效率高和反 应步骤可控的优点引起了研究者们的广泛关注。此 外, 太阳能、风能等清洁能源可以为电化学 $\mathrm{CO}_{2}$ 转 化技术提供电能, 为其大规模的工业应用提供了 广阔的前景 $11,13,14$ 。

电化学 $\mathrm{CO}_{2}$ 还原反应 $\left(\mathrm{E}-\mathrm{CO}_{2} \mathrm{RR}\right)$ 是一个包含 多质子和电子转移的复杂过程(图 1a) ${ }^{15}$, 如何实现 对于特定产物的可控催化成为当前 $\mathrm{E}-\mathrm{CO}_{2} \mathrm{RR}$ 的研 究重点。为了加快 $\mathrm{E}-\mathrm{CO}_{2} \mathrm{RR}$ 的速率, 同时使得反应 选择性地生成目标产物, 研究者们开发了众多高 活性、高选择性的E- $\mathrm{CO}_{2} \mathrm{RR}$ 催化剂。通过精细调控
催化剂的电子结构, 如构筑缺陷 ${ }^{16-18}$ 、空位 ${ }^{19-21}$ 、 合金 22,23 、特定晶界 ${ }^{24-27}$ 和晶面 ${ }^{28-30}$, 可以实现对中 间产物吸脱附过程的有效控制, 进而促进催化活 性和选择性。然而, 通过改变电子结构调控催化剂 本征活性时, 不同中间产物(如 ${ }^{*} \mathrm{CO} 、{ }^{*} \mathrm{COH} 、{ }^{*} \mathrm{CHOH}$ 和*CHO等)在催化剂表面的吸附行为存在线性关 联, 加强其中一种中间产物的吸附, 必然伴随着其 他中间产物的吸附增强, 难以实现 $\mathrm{E}-\mathrm{CO}_{2} \mathrm{RR}$ 整体 性能增强(图1b) ${ }^{31,32}$ 。除了电子结构调控, 表界面 微环境调控也可以显著影响催化剂的E- $\mathrm{CO}_{2} \mathrm{RR}$ 性 能。例如, 催化剂的微纳结构精细控制可以改变其 表面的 $\mathrm{CO}_{2}$ 浓度、催化剂-电解液界面的 $\mathrm{pH}$ 以及反 应物和产物传质过程等, 进而有望改善 E- $\mathrm{CO}_{2} \mathrm{RR}$ 催化活性、选择性和稳定性等。此外, 该类特性往 往不受线性关联的制约, 在 $E-\mathrm{CO}_{2} \mathrm{RR}$ 领域具有重 要的研究价值 ${ }^{33}$ 。

然而, 在传统涂敷催化电极制备过程中, 需要 将催化剂与粘结剂(如Nafion、聚偏氟乙烯、聚四 氟乙烯等)混合均匀后涂覆在集流体上组成功能 电极。粘结剂的使用不仅会造成催化剂团聚, 还会

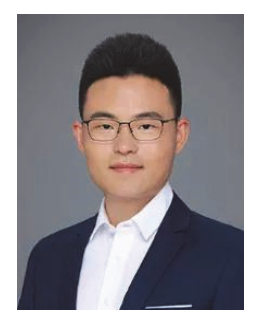

郡明飞，2014年于北京化工大学获得博 士学位, 期间于2013年获国家公派在牛 津大学化学系博士联合培养。现任北京 化工大学教授, 博士生导师。主要致力 于多维度能源材料设计与合成方法学、 新型绿色光电催化化学、结构化电极组 装与先进储能器件等研究。 
(a)

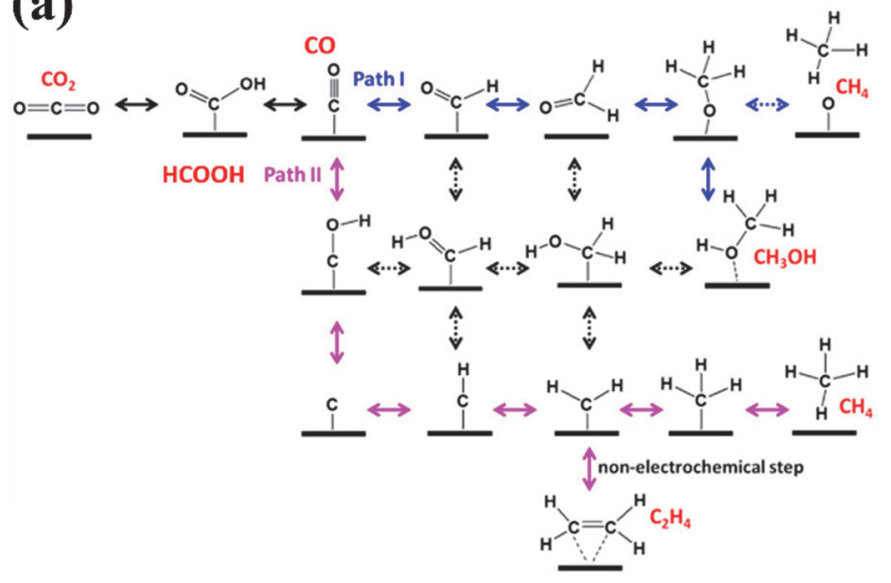

(b)

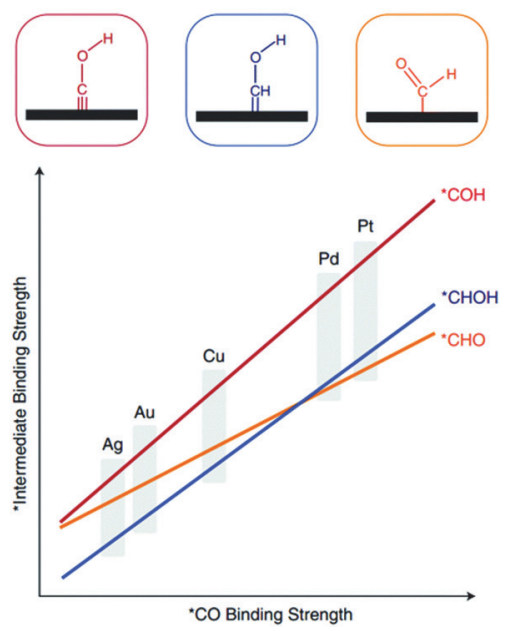

图 1 (a) $\mathrm{E}-\mathrm{CO}_{2} \mathrm{RR}$ 不同产物的反应路径 ${ }^{15}$; (b)催化剂表面 ${ }^{*} \mathrm{CO}$ 与 ${ }^{*} \mathrm{COH} 、{ }^{*} \mathrm{CHOH}$ 和 ${ }^{*} \mathrm{CHO}$ 吸附能的 函数关系的计算结果 ${ }^{31}$

Fig. 1 (a) E-CO $\mathrm{O}_{2} \mathrm{RR}$ pathways to different products ${ }^{15}$; (b) calculation results of the functional relationship between * $\mathrm{CO}$ and * $\mathrm{COH}, * \mathrm{CHOH}$ and $* \mathrm{CHO}$ adsorption energies on catalyst surface ${ }^{31}$.

Adapted from American Chemical Society and Nature Publishing Group, respectively.

阻碍电解液与活性位点的接触, 降低活性位利用 率和反应效率(图2a); 此外, 苛刻的电解液环境或 持续运行会致使粘结剂分解和催化剂脱落, 导致 电极稳定性变差, 严重制约其实际应用 $34-36$ 。因此, 如何提高电极的活性位利用率和稳定性对于高效 催化E- $\mathrm{CO}_{2} \mathrm{RR}$ 至关重要。将催化剂原位有序化(如 纳米薄膜、纳米线、纳米柱、纳米片、多级结构阵 列等)组装于集流体上得到的一体化电极可避免 粘结剂的使用, 在 E- $\mathrm{CO}_{2} \mathrm{RR}$ 中表现出诸多优势 (以 纳米柱为例, 图 2b): (1)一体化电极可以有效调控 三相界面处的微观反应环境 (如pH、反应物及反 应中间体的浓度等), 有望打破线性关联约束、实 现多种中间产物的最优吸附 ${ }^{37}$; (2)催化剂原位生 长在集流体上可以加速电子在活性位和集流体之 间转移; (3)独立分散的结构单元可以提供更大的 电化学比表面积, 暴露更多的活性位点, 确保电解 液与活性位点的充分接触; (4)结构单元之间的空 间也为电解液的传输提供了有效途径; (5)一体化 结构可以增强活性材料与集流体之间的结合强 度, 提高电极循环稳定性 ${ }^{36,38,39}$ 。因此, 设计一体 化电极对 $\mathrm{E}-\mathrm{CO}_{2} \mathrm{RR}$ 的发展具有重要研究意义。

近年来, 研究者们在不同基底(泡沫镍、泡沫 铜、导电玻璃、镍片、钛片、碳布、碳纸、碳纤维 等)上通过水/溶剂热法 40,41 、电沉积法 $42,43 、 气$ 相沉 积法 ${ }^{44,45}$ 和模板法 46,47 等方法实现了催化剂的有序 生长 ${ }^{34,35}$ (图3), 并将其直接用于 $\mathrm{E}-\mathrm{CO}_{2} \mathrm{RR}$ 的工作 电极。水/溶剂热法是制备金属氧化物和氢氧化物/ 氢氧化物等无机催化材料的有效方法。在水/溶剂
热反应中, 基底上的表面缺陷和羟基是活性物质 成核和生长的中心，从而使得纳米结构在基底上 均匀沉积 ${ }^{34,36}$ 。Guan等 ${ }^{48}$ 采用水热法在泡沫 $\mathrm{Cu}$ 上生 长了 $\mathrm{Bi}$ 掺杂的 $\mathrm{SnO}$ 纳米片 $(\mathrm{Bi}-\mathrm{SnO} / \mathrm{Cu}$ foam), 并将 其直接作为 $\mathrm{E}-\mathrm{CO}_{2} \mathrm{RR}$ 合成甲酸 $(\mathrm{HCOOH})$ 的电极。在 $-1.7 \mathrm{~V} v s . \mathrm{Ag} / \mathrm{AgCl}$ 下, 其 $\mathrm{HCOOH}$ 的法拉第效率 (Faradaic efficiency, FE) 为 $93 \%$, 电流密度为 -12 $\mathrm{mA} \cdot \mathrm{cm}^{-2}$, 能够稳定运行至少 $30 \mathrm{~h}$ 。实验结果表明 $\mathrm{Bi}$ 的掺杂能够稳定电催化剂表面二价锡 $\left(\mathrm{Sn}^{2+}\right)$ 的 存在, 使其在电化学还原过程中不易被还原为金 属 $\mathrm{Sn}\left(\mathrm{Sn}^{0}\right)$ 。此外, 结合理论计算发现, $\mathrm{Bi}$ 的掺杂 和电子从催化剂转移到 $\mathrm{Cu}$ 泡沫基底可以增强 * OCHO 中间体的吸附, 从而有利于 $\mathrm{HCOOH}$ 的生 成。电沉积法是另一种制备一体化电极的常用方 法。电沉积过程一般在选定导电基底作为工作电 极的三电极电化学池中进行, 催化材料在外加电 场下可以均匀快速地沉积。Jiao等 49 通过电沉积法 合成了具有高活性的 $\mathrm{Zn}$ 枝晶催化电极, 其 $\mathrm{CO}$ 转化 电流密度为4-14 $\mathrm{mA} \cdot \mathrm{cm}^{-2}, \mathrm{FE}$ 为 $80 \%$, 远高于体块 的 Zn催化剂 $\left(0.3-2.8 \mathrm{~mA} \cdot \mathrm{cm}^{-2}, 20 \%\right)$ 。这表明一体 化电极的设计可以显著提升 $\mathrm{E}-\mathrm{CO}_{2} \mathrm{RR}$ 性能。气相 沉积法已被广泛应用于合成一体化电极。其又可 分为物理气相沉积法和化学气相沉积法, 二者的 区别在于是否发生化学反应。Kim等 ${ }^{50}$ 采用微波辅 助化学气相沉积技术制备了含 $\mathrm{N}$ 的金刚石薄膜电 极, 通过改变生长条件控制 $s p^{2}-\mathrm{C}$ (石墨) 和 $s p^{3}-\mathrm{C}$ (金刚石)的比例, 实现对 $\mathrm{E}-\mathrm{CO}_{2} \mathrm{RR}$ 活性和选择性的 调控。 

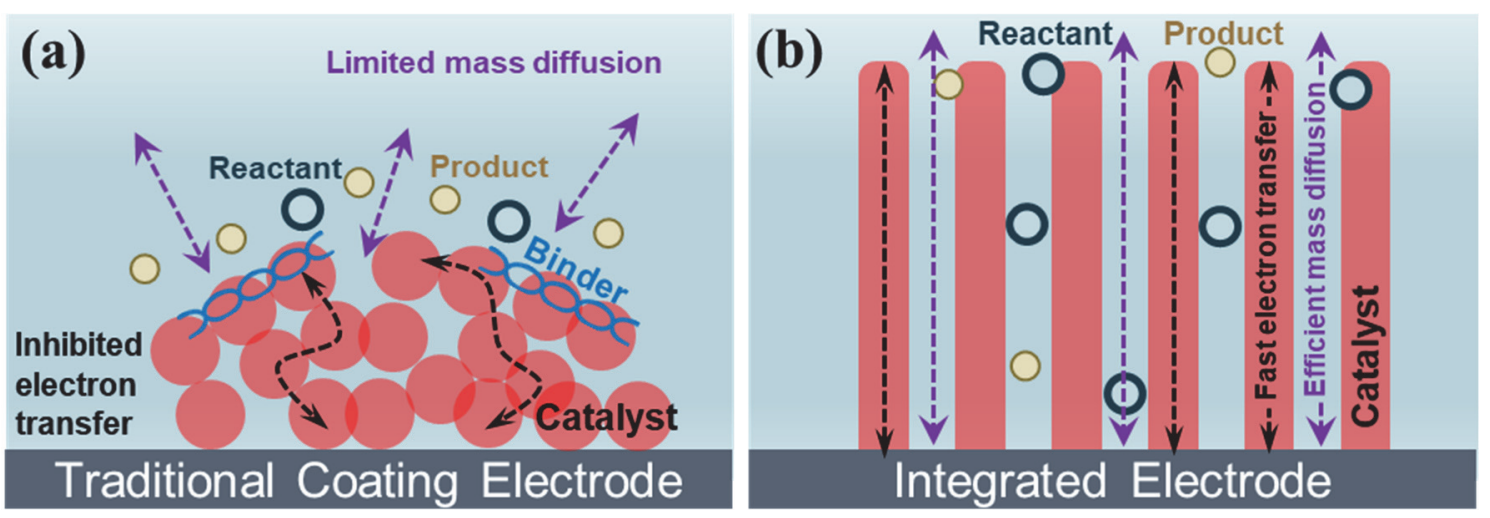

图 2 (a)传统涂敷电极和(b)一体化电极示意图

Fig. 2 Schematic illustration of (a) traditional coating electrode and (b) integrated electrode.

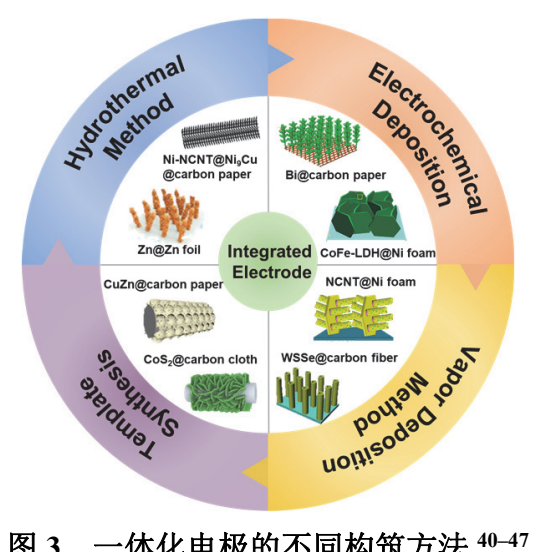

图 3 一体化电极的不同构筑方法 40-47

Fig. 3 Different construction methods of integrated electrode ${ }^{40-47}$.

Adapted from Elsevier ${ }^{40,43,45,47}$, Wiley ${ }^{41,46}$, Royal Society of Chemistry ${ }^{42}$ and American Chemical Society ${ }^{44}$ publications.

随着 $\mathrm{E}-\mathrm{CO}_{2} \mathrm{RR}$ 相关的基础研究和应用研究不 断展开, 如何从催化剂设计拓展到电极构筑, 以及 简化电极构筑方法受到研究者的广泛关注。但是, 目前关于E-CO $\mathrm{CO}_{2} \mathrm{RR}$ 的综述多集中在催化剂本征活 性调控方面, 从电极角度出发的探讨和综述鲜有 报道。基于此, 本文针对近年来 $\mathrm{E}-\mathrm{CO}_{2} \mathrm{RR}$ 领域中一 体化电极(包括一体化金属基、一体化金属化合物 基和一体化金属单原子基 $\mathrm{E}-\mathrm{CO}_{2} \mathrm{RR}$ 电极)的应用进 行了综述。详细介绍了不同一体化电极的设计思 路, 以及结构和表界面调控对 $\mathrm{E}-\mathrm{CO}_{2} \mathrm{RR}$ 性能影响 规律, 重点探讨了一体化电极对 $\mathrm{E}-\mathrm{CO}_{2} \mathrm{RR}$ 的构效 关系以及性能提升机制等。最后, 在现有研究成果 的基础上, 对一体化电极在 $\mathrm{E}-\mathrm{CO}_{2} \mathrm{RR}$ 领域的挑战 和前景进行了展望。

\section{2 一体化金属基 $\mathrm{E}-\mathrm{CO}_{2} \mathrm{RR}$ 电极}

金属是研究最早的 E- $\mathrm{CO}_{2} \mathrm{RR}$ 电催化剂之一。 1980 s 到1990s之间, Hori等 ${ }^{51-53}$ 报道了一系列具有
开创性的工作, 将金属直接用作电极在 $\mathrm{KHCO}_{3}$ 水 系电解质中催化 $\mathrm{E}-\mathrm{CO}_{2} \mathrm{RR}$ 得到一氧化碳 $(\mathrm{CO})$ 、甲 烷 $\left(\mathrm{CH}_{4}\right)$ 、甲酸盐 $\left(\mathrm{HCOO}^{-}\right)$和其他碳氢化合物。根 据还原产物的不同，将这些金属分为四类(图4a): 第一类是 $\mathrm{Au} 、 \mathrm{Ag} 、 \mathrm{Zn} 、 \mathrm{Pd}$ 和 $\mathrm{Ga}$ 等。它们能够结合 $\mathrm{CO}_{2}^{--}$中间体, 催化 $\mathrm{CO}_{2}$ 的 $\mathrm{C}-\mathrm{O}$ 键断裂, 而且 $\mathrm{CO}$ 作 为主要的还原产物, 很容易从电极表面脱附。第二 类是 $\mathrm{Sn} 、 \mathrm{~Pb} 、 \mathrm{Bi}$ 和In等。它们很难吸附 $\mathrm{CO}_{2}^{-}$中间体, 解吸的 $\mathrm{CO}_{2}^{-}$趋向于在碳原子上质子化, 最终还原

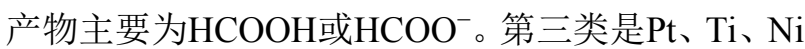
和 $\mathrm{Fe}$ 等。它们具有较低的析氢反应(HER)过电位和 较强的 $\mathrm{CO}$ 吸附能, 从而使 $\mathrm{H}_{2}$ 成为主要产物。第四 类是 $\mathrm{Cu}$ 。在目前报道的众多金属中, $\mathrm{Cu}$ 是唯一可 以 $\mathrm{E}-\mathrm{CO}_{2} \mathrm{RR}$ 生成 $\mathrm{C}_{1}-\mathrm{C}_{3}$ 产物的金属。吸附在 $\mathrm{Cu}$ 表 面的* $\mathrm{CO}$ 倾向于在高过电位下质子化生成 ${ }^{*} \mathrm{COH}$ 或* $\mathrm{CHO}$ 中间体, 进一步还原生成碳氢化合物或者 醇类物质 $7,54-56$ 。

$\mathrm{Pd}$ 在极低过电位下可催化 $\mathrm{E}-\mathrm{CO}_{2} \mathrm{RR}$ 生成 $\mathrm{HCOO}^{-}$。Zou等 57 采用脉冲电沉积的方法将介孔 $\mathrm{Pd}$ (平均孔径为 $10 \mathrm{~nm}$, 壁厚 $4 \mathrm{~nm}$ ) 沉积在高度有序 的 $\mathrm{TiO}_{2}$ 纳米管阵列上, 得到了一种新颖的多级结构 电极 $(\mathrm{mPd} / \mathrm{TNTAs}) 。-0.1 \mathrm{~V} v \mathrm{~s}$. RHE下, $\mathrm{mPd} / \mathrm{TNTAs}$ 催化E- $\mathrm{CO}_{2} \mathrm{RR}$ 至 $\mathrm{HCOO}^{-}$的FE为 $88 \% \pm 2 \%$, 比不 含介孔的 $\mathrm{Pd} / \mathrm{TNTAs}$ 高2.4倍 $(35 \% \pm 7 \%)$, 这得益于 $\mathrm{mPd} / \mathrm{TNTAs}$ 中泡沫状Pd的介孔结构促进了活性位 暴露和电解液传输。此外, 作者通过调控纳米管阵 列的结构, 实现了对纳米管内外表面 $\mathrm{CO}_{2}$ 浓度的调 节, 即一体化电极可以有效调控三相界面处的微 观反应环境(反应物及反应中间体的浓度), 实现多 种中间产物的最优吸附, 进而强化了 $\mathrm{mPd} / \mathrm{TNTAs}$ 的E- $\mathrm{CO}_{2} \mathrm{RR}$ 至 $\mathrm{HCOO}^{-}$电催化活性, 该工作所提出 的 “结构影响传质” 思想对于结构化电催化剂的设 计具有重要借鉴意义。在众多金属催化剂中，Zn 由于具有储量丰富、价格低廉等优点, 受到广泛研 
(a)

\begin{tabular}{|c|c|c|c|c|c|c|c|c|c|c|c|}
\multicolumn{1}{|c}{ CO } & \multicolumn{1}{c}{ HCOO- $^{-}$} & \multicolumn{1}{c}{$\mathrm{C}_{1}-\mathrm{C}_{3}$ product } & \multicolumn{3}{c|}{$\mathrm{H}_{2}$} \\
\hline Ti & V & Cr & Mn & Fe & Co & Ni & Cu & Zn & Ga & Ge & As \\
\hline Zr & Nb & Mo & Te & Ru & Rh & Pd & Ag & Cd & In & Sn & Sb \\
\hline Hf & Ta & W & Re & Os & Ir & Pt & Au & Hg & Tl & Pb & Bi \\
\hline
\end{tabular}

(b)

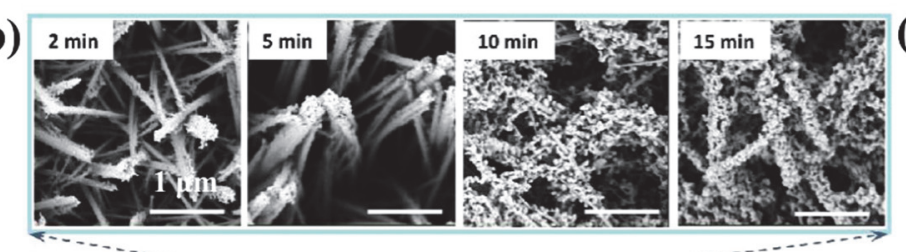

(d)
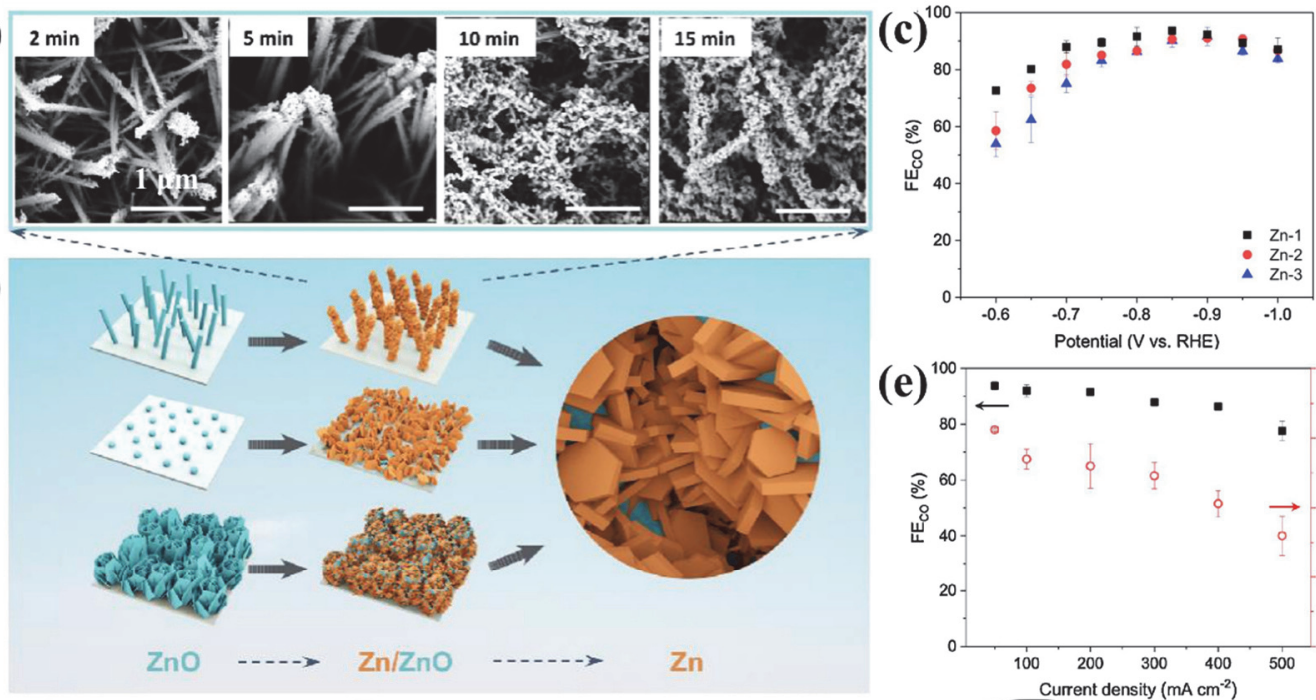

(f)

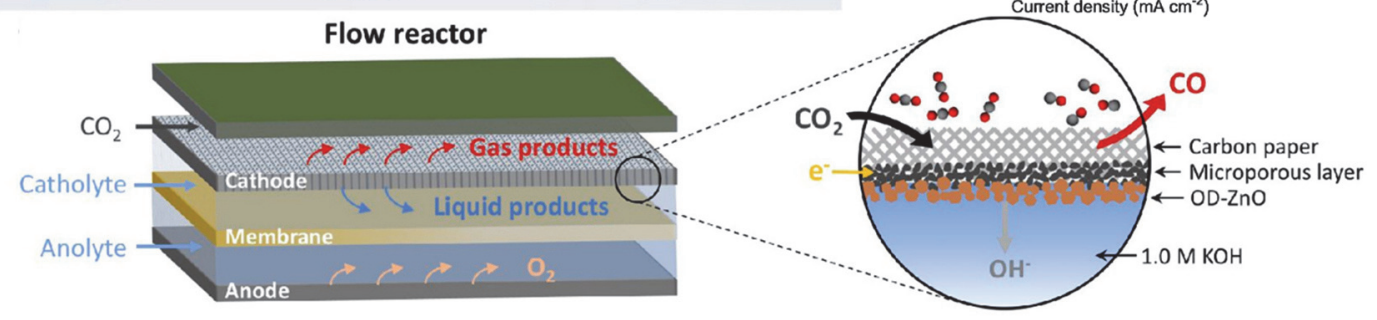

图 4 (a) E-COO $\mathrm{CO}_{2} \mathrm{RR}$ 金属分类; (b) $\mathrm{CO}_{2}$ 饱和的 $0.1 \mathrm{~mol} \cdot \mathrm{L}^{-1} \mathrm{KHCO}_{3}$ 中-1.6 Vvs. RHE 时不同还原时间下 $\mathrm{ZnO}-1$ 的 SEM 图像; (c)三个 $\mathrm{ZnO}$ 样品电化学重构过程的示意图; (d) $0.1 \mathrm{~mol} \cdot \mathrm{L}^{-1} \mathrm{KHCO}_{3}$ 中 $\mathrm{Zn}-1, \mathrm{Zn}-2$ 和 $\mathrm{Zn}-3$ 对 $\mathrm{CO}$ 的 FE; (e) $1.0 \mathrm{~mol} \cdot \mathrm{L}^{-1} \mathrm{KOH}$, 液流电池中 $\mathrm{CO}$ 的 $\mathrm{FE}$ 和阴极电压与电流密度之间的函数关系图; (f)基于 $\mathrm{OD}-\mathrm{ZnO}$ 的 气体扩散电极和液流电池的示意图 40

Fig. 4 (a) E-CO $\mathrm{O}_{2} \mathrm{RR}$ metal classification; (b) SEM images of $\mathrm{ZnO}-1$ sample reduced for different time at $-1.6 \mathrm{~V}$ vs. RHE in $\mathrm{CO}_{2}$-saturated $0.1 \mathrm{~mol} \cdot \mathrm{L}^{-1} \mathrm{KHCO}_{3}$; (c) schematic illustration of the electrochemical reconstruction process for three $\mathrm{ZnO}$ samples; (d) $\mathrm{CO} \mathrm{FE}$ of $\mathrm{Zn}-1, \mathrm{Zn}-2$ and $\mathrm{Zn}-3$ in $0.1 \mathrm{~mol} \cdot \mathrm{L}^{-1} \mathrm{KHCO}_{3}$; (e) $\mathrm{FE}$ for $\mathrm{CO}$ and cathode potential as a function of current density using $1.0 \mathrm{~mol} \cdot \mathrm{L}^{-1} \mathrm{KOH}$ in flow cell; (f) illustration of the flow cell and the OD-ZnO based gas diffusion electrode ${ }^{40}$.

Adapted from Elsevier.

究。Luo等 ${ }^{40}$ 通过 $\mathrm{ZnO}$ 的重构衍生制备了不同形貌 的Zn电极(图4b-f)。原位和非原位表征手段表明, 和 $\mathrm{Zn}$ 纳米颗粒构成的传统涂敷电极相比, 一体化 $\mathrm{Zn}$ 电极表现出显著提高的比表面积和电化学活性 比表面积。从而在催化E- $\mathrm{CO}_{2} \mathrm{RR}$ 时, 表现出优异的 选择性和稳定性, 其产 $\mathrm{CO}$ 的法拉第效率超过了 $90 \%$, 稳定性超过 $18 \mathrm{~h}$ 。将其应用于液流电池中, $\mathrm{Zn}$ 电极的FE为 $91.6 \%$, 在 $-0.62 \mathrm{~V} v s$. RHE (可逆氢 电极, reversible hydrogen electrode)下电流密度可 高达 $200 \mathrm{~mA} \cdot \mathrm{cm}^{-2}$ 。Bi具有无毒且成本较低的优点,
同时在电催化过程中可以很好地抑制 HER 的发 生, 被认为是一种很有前途的 $\mathrm{E}-\mathrm{CO}_{2} \mathrm{RR}$ 电极材 料 $42,58-61$ 。Lee等 62 采用脉冲电沉积法在 $\mathrm{Cu}$ 基底上 原位生长 $\mathrm{Bi}$ 纳米薄片。与传统的直流电沉积得到 的Bi膜相比, Bi纳米薄片具有大量的边和角活性 位点。数值模拟表明, 纳米结构的边或角位点形成 了很强的局部电场, 增强了其在水溶液中 E$\mathrm{CO}_{2} \mathrm{RR}$ 活性。 $\mathrm{Bi}$ 纳米薄片在低电位 $-0.4 \mathrm{~V} v s$. RHE 下表现出较高的 $\mathrm{HCOO}^{-}$选择性 $(\mathrm{FE}=79.5 \%),-0.6$ $\mathrm{V} v s$. RHE时其FE接近 $100 \%$, 表明形貌调控是提高 
E- $\mathrm{CO}_{2} \mathrm{RR}$ 催化效率的有效途径。此外, 一体化结构 扩大了三相反应界面并提高了电极表面 $\mathrm{CO}_{2}$ 浓 度, 实现催化性能的强化。Sun等 ${ }^{63}$ 首先在 $\mathrm{Cu}$ 箔表 面生长 $\mathrm{Cu}(\mathrm{OH})_{2}$ 纳米线, 之后进行热解、电化学还 原得到了高密度 $\mathrm{Cu}$ 纳米线 $(\mathrm{Cu} \mathrm{NW})$ 阵列电极。经 过聚四氟乙烯(PTFE)表面改性后, Cu NW阵列电 极具有 “亲气疏水” 的表面特性 $\left(\mathrm{CO}_{2}\right.$ 气泡接触角为 $48.4^{\circ}$, 电解液接触角为 $\left.136.6^{\circ}\right), \mathrm{C}_{1}$ 产物 $\mathrm{CO}$ 和 $\mathrm{HCOOH}$ 的FE增大 (分别约为 $25 \%$ 和 $26 \%$ ), 所有液 态产物的FE可达 $67 \%$ 。此外, PTFE表面改性后大 大抑制了 HER的发生 $(\mathrm{FE}<20 \%)$, 是无PTFE修饰 $\mathrm{Cu}$ 纳米线的一半。

迄今为止, 大部分关于 $\mathrm{E}-\mathrm{CO}_{2} \mathrm{RR}$ 的研究都是 在 $\mathrm{CO}_{2}$ 饱和的水溶液中进行(图5a)。但是, 室温条 件下, $\mathrm{CO}_{2}$ 在水中的溶解度较低 $\left(34 \mathrm{mmol} \cdot \mathrm{L}^{-1}\right)$, 通 过水溶液扩散到电极表面的 $\mathrm{CO}_{2}$ 有限, 使得电流密 度仅为几十 $\mathrm{mA} \cdot \mathrm{cm}^{-2}$, 远低于工业应用所需要的基 准 $\left(>300 \mathrm{~mA} \cdot \mathrm{cm}^{-2}\right)^{64}$ 。通过采用有机电解液、改变 电解液压力和温度可以提高 $\mathrm{CO}_{2}$ 的溶解度和催化 效率, 但上述策略无疑会增加催化成本和工艺复 杂性, 不利于工业化发展 ${ }^{31}$ 。现阶段, $\mathrm{H}$ 型电解池 被广泛应用于 $\mathrm{E}-\mathrm{CO}_{2} \mathrm{RR}$ 机理的研究, 但其电流密 度受到水溶液中 $\mathrm{CO}_{2}$ 溶解度较低和扩散速度较慢 的限制, 很难满足工业应用的需求 ${ }^{65,66}$ 。近期研究 表明, 通过构建带有气体扩散电极(gas diffusion electrode, GDE)的液流电池可以显著促进 $\mathrm{CO}_{2}$ 的扩 散传输(图 $5 b$ ), 其扩散路径仅约为 $50 \mathrm{~nm}$, 远低于 普通 $\mathrm{H}$ 型电解池中的扩散路径 $(50 \mu \mathrm{m}){ }^{67}$ 。气体扩 散电极由一侧负载催化剂的气体扩散层组成, 在 催化过程中, $\mathrm{CO}_{2}$ 通过气体扩散层直接扩散到催化 剂表面, E- $\mathrm{CO}_{2} \mathrm{RR}$ 发生在气-液-固三相界面处。液 流电池反应槽中阴极的电解液不断循环, 可以加 速反应物扩散吸附到催化剂表面和产物从催化剂 表面扩散脱除68。此外, 利用液流电池反应槽可以 在碱性、高 $\mathrm{pH}$ 的电解质中进行 $\mathrm{E}-\mathrm{CO}_{2} \mathrm{RR}$, 这也进 一步抑制了竞争反应HER的发生 ${ }^{67}$ 。一体化电极在 构筑气体扩散电极方面具有明显优势, Sargent等 69 采用电化学抛光的 $\mathrm{Cu}$ 片作为阴极催化剂在 $\mathrm{H}$ 型电 解池中测试了其 $\mathrm{E}-\mathrm{CO}_{2} \mathrm{RR}$ 性能, 产 $\mathrm{CH}_{4}$ 的 $\mathrm{FE}$ 和电 流密度仅为 $49 \%$ 和 $9 \mathrm{~mA} \cdot \mathrm{cm}^{-2}$ (图 $5 \mathrm{c}-\mathrm{e}$ )。而在液流电 池中, 将流动池中局部 $\mathrm{pH}$ 值与 $\mathrm{H}$ 型电解池相匹配 后, $\mathrm{Cu}$ 催化剂催化产 $\mathrm{CH}_{4}$ 的 $\mathrm{FE}$ 可维持在 $48 \% \pm 4 \%$, 但其电流密度提高至 $120 \pm 10 \mathrm{~mA} \cdot \mathrm{cm}^{-2}$, 远大于 $\mathrm{H}$ 型电解池中的电流密度, 阴极能量效率可达 $23 \%$ 。 此外, 优化后的液流电池系统在 $250 \mathrm{~mA} \cdot \mathrm{cm}^{-2}$ 的电 流密度下稳定运行 $14 \mathrm{~h}$, 同时维持 $\mathrm{CH}_{4}$ 的FE在 $40 \%$ 以上。

由于 $\mathrm{E}-\mathrm{CO}_{2} \mathrm{RR}$ 反应路径和产物繁多，用单一 金属电极调节 $\mathrm{E}-\mathrm{CO}_{2} \mathrm{RR}$ 的选择性并抑制副反应 HER的发生仍然是一个挑战。将两种金属合金化
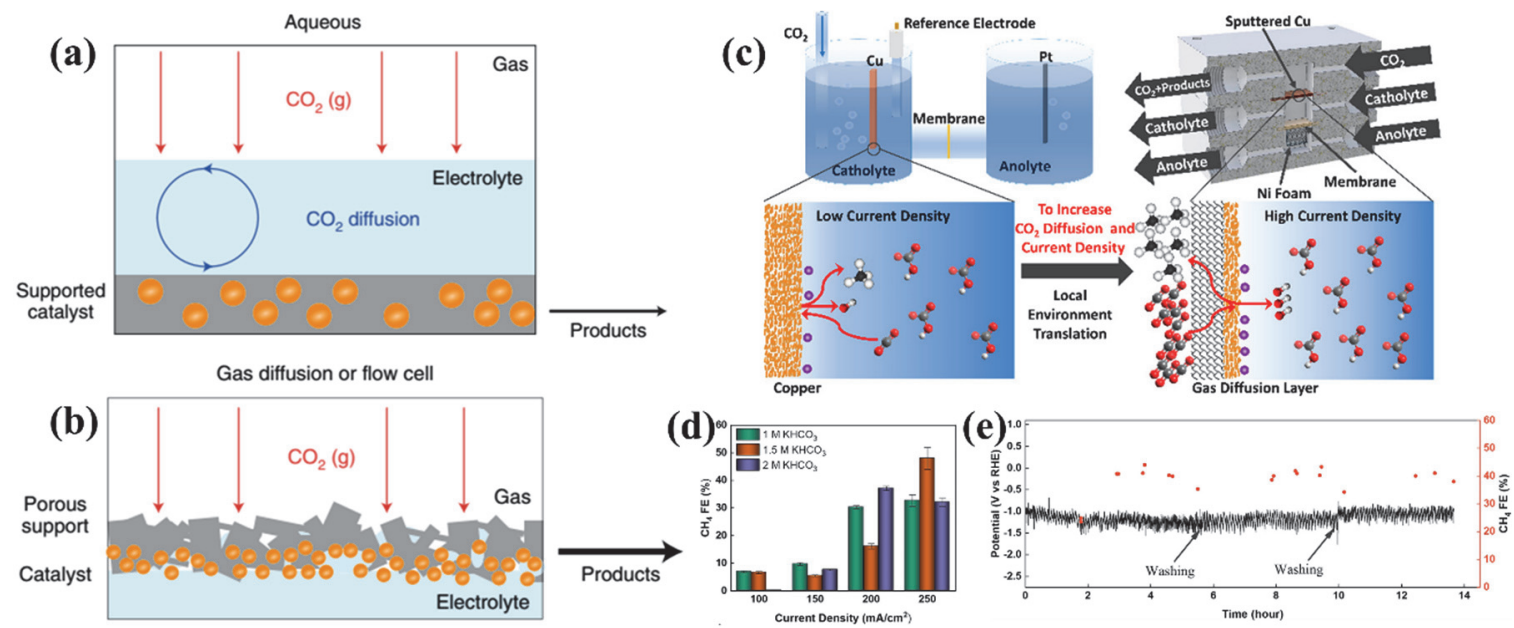

图 $5 \mathrm{E}-\mathrm{CO}_{2} \mathrm{RR}$ 溶液的动力学(a) $\mathrm{CO}_{2}$ 在水溶液中的扩散与溶解; (b) $\mathrm{CO}_{2}$ 在流动池活性层上直接溶解 ${ }^{31}$;

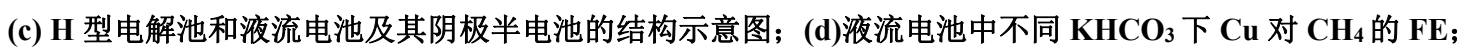
(e) $\mathrm{Cu}$ 在液流电池 $1.5 \mathrm{~mol} \cdot \mathrm{L}^{-1} \mathrm{KHCO}_{3}, 250 \mathrm{~mA} \cdot \mathrm{cm}^{-2}$ 下运行 $14 \mathrm{~h}^{69}$

Fig. 5 Solution dynamics in E-CO2 $\mathrm{CR}_{2}$ (a) $\mathrm{CO}_{2}$ dissolution and diffusion in an aqueous system; (b) direct dissolution of $\mathrm{CO}_{2}$ at a thin active layer in a flow cell ${ }^{31}$; (c) schematic of the $\mathrm{H}$-cell and flow cell configuration and their cathodic half-cell configurations; (d) $\mathrm{CH}_{4} \mathrm{FE}$ on $\mathrm{Cu}$ with different $\mathrm{KHCO}_{3}$ concentrations in flow cells; (e) $14 \mathrm{~h}$ study of $\mathrm{Cu}$ by applying $250 \mathrm{~mA} \cdot \mathrm{cm}^{-2}$ with a $1.5 \mathrm{~mol} \cdot \mathrm{L}^{-1} \mathrm{KHCO}_{3}$ electrolyte in a flow cell ${ }^{69}$.

Adapted from Nature Publishing Group ${ }^{31}$ and American Chemical Society ${ }^{69}$, respectively. 
是提升 $\mathrm{E}-\mathrm{CO}_{2} \mathrm{RR}$ 性能的有效途径之一。通过精细 调控合金表面的组成和电子结构, 能够改变反应 中间体在电极表面的吸附情况 $22,65,70,71$ 。An等 ${ }^{72}$ 通 过在温和条件下对 $\mathrm{Au}_{20} \mathrm{Cu}_{80}$ 合金进行可控的氧化 腐蚀, 合成了导电性和柔韧性良好的表面富 $\mathrm{Au}$ 的 一体化纳米多孔 $\mathrm{Au}_{3} \mathrm{Cu}$ 电极。在低电势 $(-0.7 \mathrm{~V} v s$. RHE)下, CO的FE高达 $98.12 \%$, 是自制的纳米多孔 $\mathrm{Au}$ 电极的 1.47 倍。 $\mathrm{Cu}$ 的掺杂不仅提高了 $\mathrm{Au}$ 对 $\mathrm{CO}$ 产 物的选择性, 还可以降低贵金属 $\mathrm{Au}$ 的用量。理论 计算表明, 纳米多孔 $\mathrm{Au}_{3} \mathrm{Cu}$ 电极对 $* \mathrm{COOH}$ 中间体 的吸附能最低, $\mathrm{CO}$ 解吸所需能量降低。 $\mathrm{Cu}$ 的引入 使得 $\mathrm{Au}$ 原子表面带有更多的负电荷, ${ }^{*} \mathrm{CO}$ 在距离 较远的纳米多孔 $\mathrm{Au}_{3} \mathrm{Cu}$ 表面形成稳定的双齿结构, 使得 $\mathrm{CO}$ 更容易从表面解吸, 并且避免了 $\mathrm{CO}$ 毒化。 实现高电流密度和提高目标产物选择性是推动 $\mathrm{E}-$ $\mathrm{CO}_{2}$ RR工业化应用的重要目标。Sargent等 ${ }^{73}$ 采用共 溅射法合成了一系列不同 $\mathrm{Ag}-\mathrm{Cu}$ 比例的 $\mathrm{Ag} / \mathrm{Cu}$ 合 金作为 GDE, 电解液为 $1 \mathrm{~mol} \cdot \mathrm{L}^{-1} \mathrm{KOH}$ 的液流电池 中, $\mathrm{Ag}_{0.14} / \mathrm{Cu}_{0.86}$ 在 $-0.67 \mathrm{~V} v$ s. RHE时电流密度可 达 $250 \mathrm{~mA} \cdot \mathrm{cm}^{-2}, \mathrm{C}_{2} \mathrm{H}_{5} \mathrm{OH}$ 的 $\mathrm{FE}$ 为 $41 \%$, 阴极能量效 率为 $25 \%$ 。作为对照, $\mathrm{Cu}$ 对 $\mathrm{C}_{2} \mathrm{H}_{5} \mathrm{OH}$ 的 $\mathrm{FE}$ 仅为 $29 \%$ 。 先前的报道表明 $\mathrm{Cu}$ 表面更容易生成 $\mathrm{C}_{2} \mathrm{H}_{4}$ 而不是 $\mathrm{C}_{2} \mathrm{H}_{5} \mathrm{OH}$, 通过引入第二种元素 $\mathrm{Ag}$, 其与碳的结合 能力不如 $\mathrm{Cu}$, 可以降低 $\mathrm{C}_{2} \mathrm{H}_{4}$ 中间体在催化剂表面 形成的可能性, 同时 $\mathrm{Ag} / \mathrm{Cu}$ 合金表面更有利于 $\mathrm{C}_{2} \mathrm{H}_{5} \mathrm{OH}$ 中间体的形成, 从而提高 $\mathrm{C}_{2} \mathrm{H}_{5} \mathrm{OH}$ 的选 择性。

\section{3 一体化金属化合物基 $\mathrm{E}-\mathrm{CO}_{2} \mathrm{RR}$ 电极}

与金属相比, 金属氧化物中金属元素价态易 调、富含丰富的界面和缺陷等特征能够提高 $\mathrm{E}$ $\mathrm{CO}_{2} \mathrm{RR}$ 电极的本征活性。甚至适当的形貌和氧化 程度可能使之前不具备 $\mathrm{CO}_{2}$ 还原催化活性的材料 变成一种高活性的E- $\mathrm{CO}_{2} \mathrm{RR}$ 电极 ${ }^{74-77}$ 。Qiu等 ${ }^{78}$ 设 计合成了泡沫碳支撑的富含氧空位的 $\mathrm{SnO}_{x}$ 纳米片 电极 $\left(\mathrm{V}_{\mathrm{O}}-\mathrm{SnO}_{x} / \mathrm{CF}-40\right)$, 在 $-1.0 \mathrm{~V} v s$. RHE时, 其 $\mathrm{HCOO}^{-}$的电流密度为 $-30 \mathrm{~mA} \cdot \mathrm{cm}^{-2}$, $\mathrm{FE}$ 为 $86 \%$ (图 $6 \mathrm{a}-\mathrm{d}) 。-1.2 \mathrm{~V} v s$. RHE时, $\mathrm{HCOO}^{-}$的电流密度可达 $-40 \mathrm{~mA} \cdot \mathrm{cm}^{-2}$, 其产率高达 $432.8 \mu \mathrm{mol} \cdot \mathrm{h}^{-1} \cdot \mathrm{cm}^{-2}$ 。由 于 $\mathrm{SnO}_{x}$ 纳米片的氧空位和三维的大孔泡沫碳基底 的存在, 使得其具有更高暴露的活性位点、更快的 电子转移/传质以及 $\mathrm{CO}_{2}$ 吸附/活化三者之间的协同 效应, 从而促进了E-CO 2 RR。Tricoli等79使用热气 溶胶合成策略在碳纤维纸上直接自组装得到了 $3 \mathrm{D}$ $\mathrm{Bi}_{2} \mathrm{O}_{3}$ 电极 (f- $-\mathrm{Bi}_{2} \mathrm{O}_{3}$ ), 具有优异的E- $\mathrm{CO}_{2} \mathrm{RR}$ 性能, 其 起始过电位低至 $-0.6 \mathrm{~V} v s$. RHE, $\mathrm{HCOO}^{-}$的 $\mathrm{FE}$ 为 $87 \%, \mathrm{HCOO}^{-}$产率为 $162 \mu \mathrm{mol} \cdot \mathrm{h}^{-1}$ 。相比于通过滴
涂法得到的 $\mathrm{Bi}_{2} \mathrm{O}_{3}$ 传统涂敷电极 $\left(-15.5 \mathrm{~mA} \cdot \mathrm{mg}^{-1}\right)$, $\mathrm{HCOO}^{-}$的部分电流密度为 $-20.9 \mathrm{~mA} \cdot \mathrm{cm}^{-2}(-52.2$ $\left.\mathrm{mA} \cdot \mathrm{mg}^{-1}\right)$, 提高了3 倍左右。结果表明, 该方法倾 向于产生暴露 $\beta$ 相的粗糙 $\mathrm{Bi}_{2} \mathrm{O}_{3} / \mathrm{Bi}$ 电极, 其边缘活 性位点的暴露和催化剂独特的多级结构是获得高 $\mathrm{E}-\mathrm{CO}_{2} \mathrm{RR}$ 活性的关键因素。此外, 原位生长在集流 体上可以加速电子在活性位和集流体之间转移, 有利于电流密度的提升。Luo等 ${ }^{80}$ 在导电碳纸上有 序化组装得到了具有中孔和丰富缺陷的超薄 $\mathrm{Bi} / \mathrm{Bi}_{2} \mathrm{O}_{3}$ 纳米阵列, 在 $-0.87 \mathrm{~V} v s$. RHE下, $\mathrm{HCOO}^{-}$ 的电流密度和 $\mathrm{FE}$ 分别为 $32.4 \mathrm{~mA} \cdot \mathrm{mg}^{-1} \cdot \mathrm{cm}^{-2}$ 和 $90.3 \%$ 。多级的介孔结构加速了电子转移, 增加了 $\mathrm{CO}_{2}$ 的吸附, 缩短了 $\mathrm{CO}_{2}$ 和电解质离子的扩散途 径。此外, $\mathrm{Bi} / \mathrm{Bi}_{2} \mathrm{O}_{3}$ 金属/氧化物结的组成和结构使 得局域的电子态有所不同, 从而协同促进了 $\mathrm{CO}_{2}$ 的 活化和质子化以及还原中间体的稳定。近年来, E$\mathrm{CO}_{2} \mathrm{RR}$ 产 $\mathrm{CH}_{4}$ 仅在较低的电流密度 $\left(<50 \mathrm{~mA} \cdot \mathrm{cm}^{-2}\right)$ 下才能实现一定的 $\mathrm{FE}(>50 \%)$, 远未达到工业化应 用的标准 ${ }^{81}$ 。采用 GDE和液流电池反应槽可以克服 $\mathrm{CO}_{2}$ 在水中溶解度较小和扩散速率较慢的限制, 实 现电流密度提高至 $100 \mathrm{~mA} \cdot \mathrm{cm}^{-2}$ 以上的稳定运行。 但是, 在液流电池系统中高电流密度会使得局部 $\mathrm{pH}$ 值升高, 降低水还原的驱动力, 并降低催化剂 表面对* $\mathrm{H}$ 的吸附, 不利于* $\mathrm{CO}$ 的质子化, 从而抑制 了 $\mathrm{CH}_{4}$ 的生成 ${ }^{82-86}$ 。Sargent等 ${ }^{87}$ 在 $\mathrm{Cu}$ 表面合成了具 有稳定配体的 $\mathrm{CoO}$ 纳米簇, 在液流电池中, 实现了 $225 \mathrm{~mA} \cdot \mathrm{cm}^{-2}$ 的电流密度和 $60 \%$ 的 $\mathrm{CH}_{4}$ 选择性, 并 且能够稳定运行 $18 \mathrm{~h}$ 。DFT结果表明, 纯 $\mathrm{Cu}$ 表面 ${ }^{*} \mathrm{CO}$ 的质子化能为 $0.43 \mathrm{eV}$, 当局部* $\mathrm{H}$ 不足时, $\mathrm{Cu}$ 更倾向于通过二聚生成 $\mathrm{C}_{2+}$ 产物。 $\mathrm{CoO}$ 纳米簇的引 入提高了局部* $\mathrm{H}$ 的可利用性, 使得 $\mathrm{Cu}$ 表面* $\mathrm{CO}$ 的 质子化更容易发生, 提升了 $\mathrm{CH}_{4}$ 的选择性。

过渡金属硫化物电极已经在HER ${ }^{88,89}$ 、有机小 分子氧化 ${ }^{90,91}$ 、光催化 ${ }^{92}$ 和超级电容器 93 等领域展 示了广泛的应用前景, 近年来在E- $\mathrm{CO}_{2} \mathrm{RR}$ 中的应 用研究与日俱增 ${ }^{94-98}$ 。Luo 等 ${ }^{99}$ 设计合成了一种泡 沫 $\mathrm{Ni}$ 支撑的 $\mathrm{CuS}$ 纳米片阵列 $(\mathrm{CuS} @ \mathrm{NF})$ 电极, 其中 $\mathrm{CuS}$ 纳米片的厚度为 $20-25 \mathrm{~nm}$ (图6e-h)。-1.1 Vvs. $\mathrm{RHE}$ 时, $\mathrm{CuS} @ \mathrm{NF}$ 电极对 $\mathrm{CH}_{4}$ 的FE为 $73 \% \pm 5 \%$, 稳定运行时间可达 $60 \mathrm{~h}$ 。除了 $\mathrm{CuS}$ 优异的E- $\mathrm{CO}_{2} \mathrm{RR}$ 本征活性, 开放的一体化纳米片阵列结构赋予了 电极高效的传质过程和较大的电化学活性面积 $\left(C_{\mathrm{dl}}=39.82 \mathrm{mF} \cdot \mathrm{cm}^{-2}\right)$ 。此外, 直接生长在泡沫镍骨 架上的 $\mathrm{CuS}$ 纳米片具有良好的机械附着力和较高 的结构稳定性。 $\mathrm{He}$ 等 ${ }^{46}$ 提出了一种由直接生长在 碳布上的 $2 \mathrm{D} \mathrm{CoS}_{2}$ 纳米片阵列和 $3 \mathrm{D} \mathrm{CoS}_{2}$ 纳米笼组 
(a)
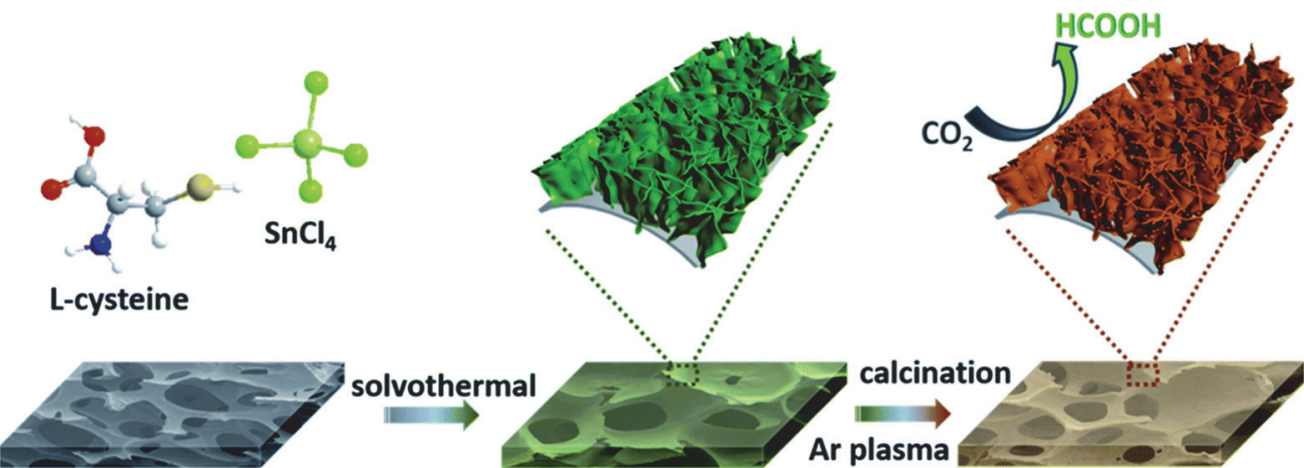

Carbon Foam olvotherm

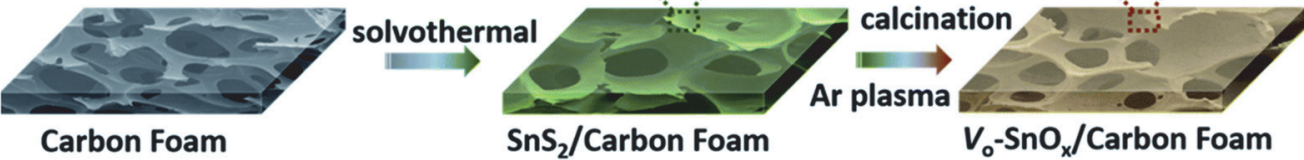
r plasma

(b)
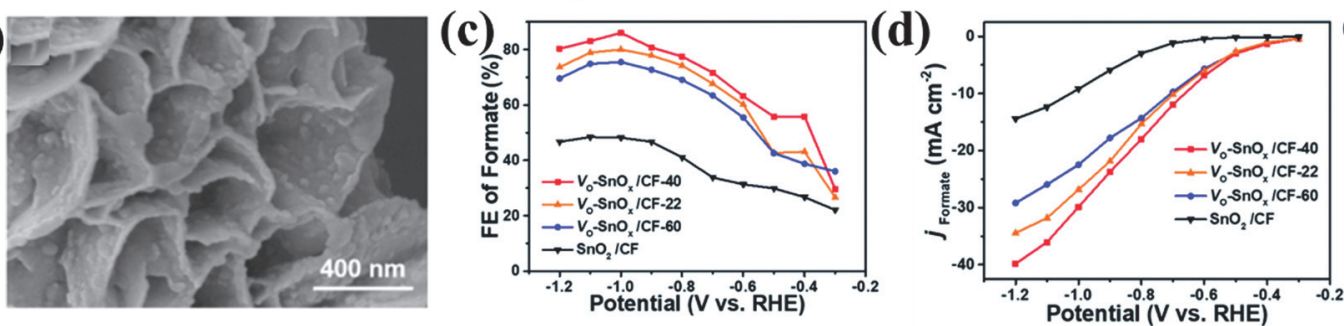

(e)
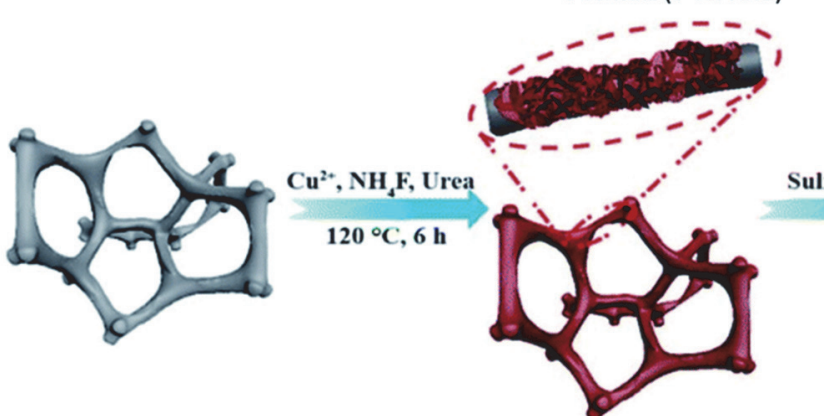

Sulfuration

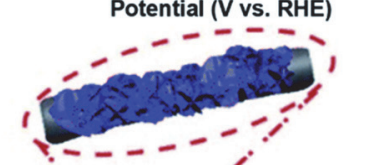

Ni foam

$\mathrm{Cu}_{2} \mathrm{O} @ \mathrm{NF}$

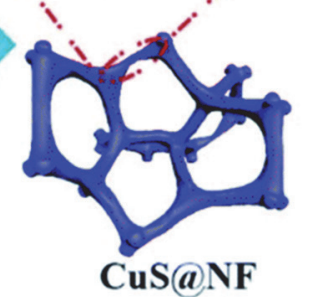

(f)
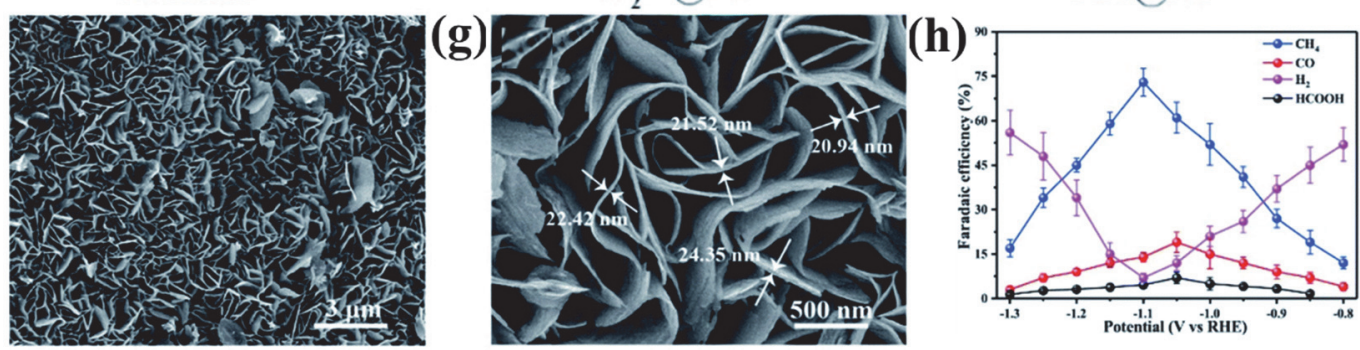

图 6 (a)碳泡沫上富含氧空位的 $\mathrm{SnO}_{x}$ 纳米片合成示意图; (b) $\mathrm{VO}_{0}-\mathrm{SnO}_{x} / \mathrm{CF}-40$ 的 $\mathrm{SEM}$ 图像; 不同电极对 $\mathrm{HCOO}^{-}$的 (c) FE 和(d) 电流密度 ${ }^{78}$; (e) CuS@NF 的合成示意图; CuS@NF 的(f)低倍和(g)高倍 SEM 图像;

(h)不同电压下 $\mathrm{CuS} @ \mathrm{NF}$ 产 $\mathrm{CH}_{4} 、 \mathrm{CO} 、 \mathrm{H}_{2}$ 和 $\mathrm{HCOOH}$ 的 $\mathrm{FE}{ }^{99}$

Fig. 6 (a) Schematic illustration of the fabrication process of the oxygen vacancy-enriched $\mathrm{SnO}_{x}$ nanosheets grown on carbon foam; (b) SEM images of $\mathrm{V}_{\mathrm{O}-\mathrm{SnO}} / \mathrm{CF}-40$; (c) $\mathrm{FE}$ of formate and (d) partial current density of $\mathrm{HCOO}^{-}$for various electrodes ${ }^{78}$; (e) illustration of the fabrication process of the CuS@NF sample; (f) and (g) low and high-magnification SEM images of the $\mathrm{CuS} @ \mathrm{NF}$; (h) $\mathrm{FE}$ for $\mathrm{CH}_{4}, \mathrm{CO}, \mathrm{H}_{2}$ and $\mathrm{HCOOH}$ at various applied potentials ${ }^{99}$. Adapted from Royal Society of Chemistry ${ }^{78,99}$.

成的多级 $\mathrm{CoS}_{2}$ 纳米笼电极。研究表明 $3 \mathrm{D} \mathrm{CoS}_{2}$ 纳米 笼/2D $\mathrm{CoS}_{2}$ 纳米片阵列的多级结构不仅可以避免 $\mathrm{CoS}_{2}$ 纳米笼的不规则叠加, 从而使活性位点充分 暴露, 还加速了电子在活性位和集流体之间转移。 $-0.6 \mathrm{~V} v s$. RHE时， $3 \mathrm{D} \mathrm{CoS}_{2}$ 纳米笼 $/ 2 \mathrm{D} \mathrm{CoS}$ 纳米片 阵列产 $\mathrm{CO}$ 的最大 $\mathrm{FE}$ 为 $85.7 \%$, 电流密度为 -2.8
$\mathrm{mA} \cdot \mathrm{cm}^{-2}$, 是粉体 $\mathrm{CoS}_{2}$ 纳米笼催化剂的3倍。此外, 一体化电极纳米片阵列之间的空间也为电解液的 传输提供了有效途径, 确保了电解液与活性位点 的充分接触。理论计算表明, $\mathrm{CoS}_{2}$ 的 $\mathrm{S}$ 平面对 $\mathrm{CO}_{2} \mathrm{RR}$ 表现出较高的活性, 而对HER活性较低; 而 $\mathrm{S}$ 边缘对HER活性高, 对 $\mathrm{CO}_{2} \mathrm{RR}$ 不利。因此, 这种 
$\mathrm{S}$ 边缘较少的多级 $\mathrm{CoS}_{2}$ 纳米笼电极大大抑制了 $\mathrm{HER}$ ，从而显著促进了 $\mathrm{E}-\mathrm{CO}_{2} \mathrm{RR}$ 的发生。

除过渡金属硫化物外, Sun等 ${ }^{100}$ 在 Ti网上合成 了 $\mathrm{FeP}$ 纳米阵列 $(\mathrm{FeP} \mathrm{NA} / \mathrm{TM})$, 直接作为高效的一 体化催化电极用于 $\mathrm{E}-\mathrm{CO}_{2} \mathrm{RR}$ 。 FeP NA/TM在 $-0.2 \mathrm{~V}$ vs. RHE 时对 $\mathrm{CH}_{3} \mathrm{OH}$ 的 $\mathrm{FE}$ 为 $80.2 \%, \mathrm{CH}_{3} \mathrm{OH}$ 和 $\mathrm{C}_{2} \mathrm{H}_{5} \mathrm{OH}$ 的总 $\mathrm{FE}$ 可达 $94.3 \%$ 。在 $36 \mathrm{~h}$ 的连续电催化 过程中, 也表现出非常高的稳定性。理论计算结果 表明相邻两个 $\mathrm{Fe}$ 原子的协同作用有利于 $\mathrm{CO}_{2}$ 转化 为 $\mathrm{CH}_{3} \mathrm{OH}$ 的吸附和还原过程。但是, 由于过渡金 属磷化物在水溶液中更倾向于发生HER, 因此在 E- $\mathrm{CO}_{2} \mathrm{RR}$ 领域报道较少 ${ }^{101}$ 。

\section{4 一体化金属单原子基E- $\mathrm{CO}_{2} \mathrm{RR}$ 电极}

碳材料具有材料来源丰富、结构易调、表面积 大、稳定性高和环境友好等明显的优势, 其多孔结 构也有利于 $\mathrm{CO}_{2}$ 的吸附和电解液的快速渗透 ${ }^{4}$ 。碳
材料负载的单原子催化剂(SACs)由于具有最大的 理论原子利用效率、可调的金属配位结构、独特的 载体效应等, 在许多电化学反应中表现出优异的 催化性能和选择性 ${ }^{102-107}$ 。He等 ${ }^{108}$ 采用静电纺丝法 设计合成了一种 $\mathrm{Ni}$ 单原子/多孔碳纤维膜电极 (NiSA/PCFM), 广泛分布在碳纳米纤维中的 $\mathrm{Ni}$ 单 原子位点对 $\mathrm{CO}_{2}$ 的活化起着决定性作用, 多孔、互 相连接的碳纳米纤维为 $\mathrm{CO}_{2}$ 扩散和电子传输提供 了重要通道(图7a-b)。相比于喷涂粉体法得到的P$\mathrm{NiSA} / \mathrm{PCFM}$ 传统涂敷电极 $\left(\mathrm{FE}_{\mathrm{CO}}=87 \%\right)$, NiSA/ PCFM一体化和多孔结构极大促进了 $\mathrm{Ni}$ 单原子的 暴露和利用, 从而确保电解液与活性位点的充分 接触, 表现出优异的催化活性 $\left(\mathrm{FE}_{\mathrm{CO}}=96 \%\right)$ 。此外, 一体化结构增强了活性材料与集流体之间的结合 强度, 提高电极循环稳定性。NiSA/PCFM一体化 电极作为 GDE应用在液流电池中时, $-1.0 \mathrm{~V} v \mathrm{~s}$. RHE下, 其CO的电流密度高达 $-308.4 \mathrm{~mA} \cdot \mathrm{cm}^{-2}, \mathrm{FE}$ (a)

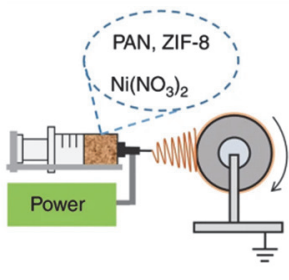

(c)

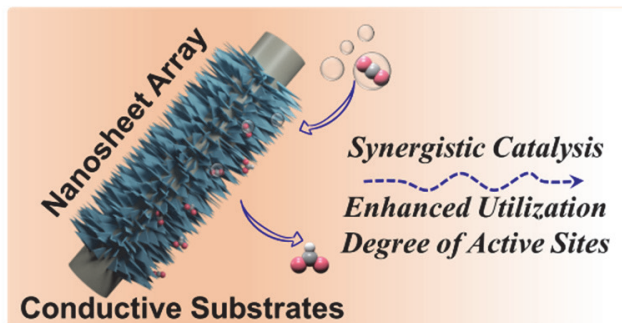

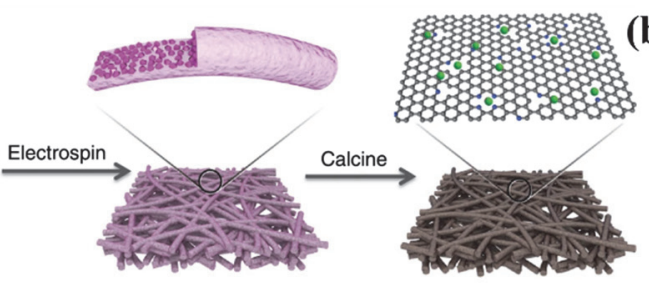

(b)
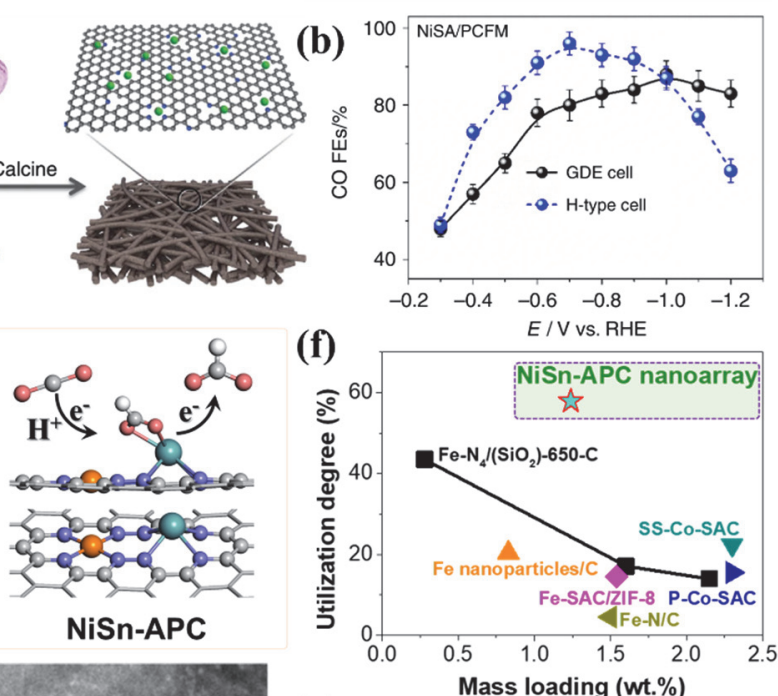

(d)
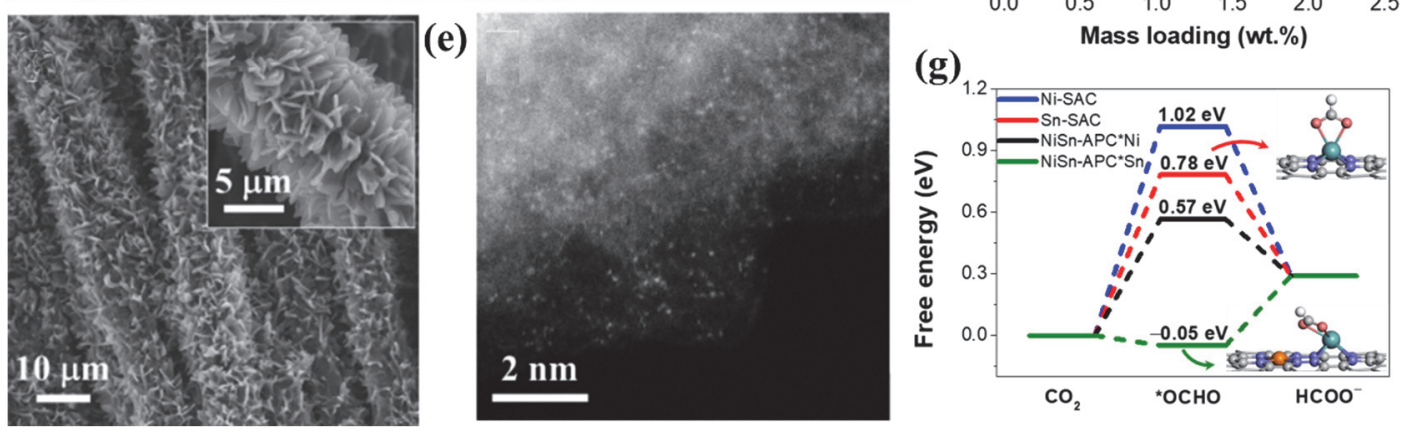

图 7 (a) NiSA/PCFM 的合成策略; (b)不同电解池中不同电压下 NiSA/PCFM 对 CO 的 FE ${ }^{108}$; (c) NiSn-APC 示意图;

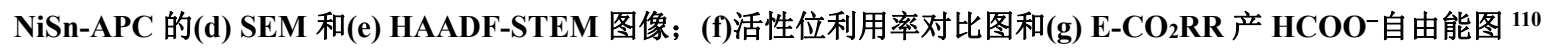

Fig. 7 (a) Synthesis strategy of NiSA/PCFM; (b) CO FE of NiSA/PCFM at various cathode potentials in different cells ${ }^{108}$; (c) scheme illustration for the integrated NiSn-APC electrode; (d) SEM images and (e) HAADF-STEM image of NiSn-APC; (f) a comparative study on utilization degree of NiSn-APC and various materials; (g) free energy diagrams in E-COO 2 RR to $\mathrm{HCOO}^{-}$on NiSn-APC and references ${ }^{110}$. 
为 $88 \%$, 能够稳定运行 $120 \mathrm{~h}$ 。 Wu等 ${ }^{109}$ 利用氮掺杂 的碳和大块状金属 $\mathrm{Ni}$ 固体之间的扩散合成了多 级、一体化和原子化的催化电极 $(\mathrm{H}-\mathrm{CPs}) 。-1.0 \mathrm{~V}$ $v s$. RHE时, H-CPs催化E- $\mathrm{CO}_{2} \mathrm{RR}$ 生成CO的FE高达 $97 \%$, 电流密度为 $-48.66 \mathrm{~mA} \cdot \mathrm{cm}^{-2}$, 稳定性超过 40 $\mathrm{h}$ 。在 $-0.7--1.2 \mathrm{~V} v s$. RHE的电势区间内, FE可维 持在 $90 \%$ 以上。此外, N-CNTs的垂直排列结构不 仅具有优良的导电性, 而且其表面的超亲水性和 超疏气性, 也为离子扩散提供了较大的接触面积。 特别是在高反应速率下, 超亲水结构有利于通过 电解液润湿催化剂表面进行传质, 而超疏气表面 可以促进气体产物的脱附。

虽然SACs在E- $\mathrm{CO}_{2} \mathrm{RR}$ 领域受到广泛关注, 但 单一SACs活性位点很难满足具有多电子/多中间 产物E-CO ${ }_{2} \mathrm{RR}$ 的最优催化 ${ }^{33}$ 。因此, 如何通过对催 化剂结构进行精细调控来提高催化效率及活性位 利用率是亟需解决的关键科学问题。我们 ${ }^{110}$ 提出 了一种负载 $\mathrm{NiSn}$ 原子对催化剂的二维碳基阵列一 体化电极 (NiSn-APC), 在E- $\mathrm{CO}_{2} \mathrm{RR}$ 产 $\mathrm{HCOO}^{-}$时表 现出协同效应, 实现了本征活性以及活性位利用 率的显著提升(图7c-g)。电化学结果表明, 所制备 的 NiSn-APC 对 E- $\mathrm{CO}_{2} \mathrm{RR}$ 产 $\mathrm{HCOO}^{-}$具有优异的催 化性能, 其 $\mathrm{HCOO}^{-}$的产率和转换频率分别达到了 $36.7 \mathrm{~mol} \cdot \mathrm{h}^{-1} \cdot \mathrm{g}^{-1}$ 和 $4752 \mathrm{~h}^{-1}$, 在目前报道的 $\mathrm{Sn}$ 基催 化剂中处于优势地位。相比于滴涂粉体法得到的 $\mathrm{NiSn}-\mathrm{APC}$ 传统涂敷电极 $\left(\mathrm{FE}_{\mathrm{HCOO}}{ }^{-}=62.4 \%\right.$, 电流密 度为 $-28.5 \mathrm{~mA} \cdot \mathrm{cm}^{-2}$ ), NiSn-APC一体化电极的FE 和电流密度大幅提升, 分别为 $86.1 \%$ 和 -43.7 $\mathrm{mA} \cdot \mathrm{cm}^{-2}$ 。一体化电极的结构优势加速了电子在活 性位和集流体之间的转移, 也为电解液的传输提 供了有效途径, 进而提升了目标产物的选择性和 电流密度。此外, 其Tafel斜率 $\left(120 \mathrm{mV} \cdot \mathrm{dec}^{-1}\right)$ 明显 小于滴涂粉体法得到的NiSn-APC传统涂敷电极 $\left(164 \mathrm{mV} \cdot \mathrm{dec}^{-1}\right)$, 表明一体化的纳米阵列结构具有 更快的反应动力学, 加速了目标产物的生成。活性 位定量研究表明, 得益于独特的多级纳米片阵列 结构, NiSn-APC表现出了高达 $57.9 \%$ 的活性位利 用率, 显著高于粉体NiSn-APC材料(32.7\%)。密度 泛函理论(DFT)计算表明 $\mathrm{Ni}^{-} \mathrm{N}_{4}$ 的掺杂有利于 $\mathrm{Sn}-$ $\mathrm{N}_{4}$ 位点对于 $\mathrm{HCOO}^{-}$路径中间产物* $\mathrm{OCHO}$ 的吸附, 显著降低了 $\mathrm{E}-\mathrm{CO}_{2} \mathrm{RR}$ 产 $\mathrm{HCOO}^{-}$的反应能垒。 $\mathrm{Ni}-\mathrm{N}_{4}$ 可使邻近 $\mathrm{Sn}$ 原子上的电子离域, 从而表现出更高 的活性。上述工作表明, 通过对多级二维碳纳米片 阵列表面催化位点进行精细设计, 实现了对材料 本征活性的可控调控, 不仅强化了电极反应过程, 还保证了活性位的超高利用率。

\section{5 总结与展望}

综上所述, 一体化电极在提升活性位利用率, 调控面处微环境以及强化反应稳定性等方面具有 重要的优势, 特别是对面向实际应用的催化反应 研究起到了重要推动作用。本文从 $\mathrm{E}-\mathrm{CO}_{2} \mathrm{RR}$ 基一 体化电极的设计方法, 最新电极种类进展(金属、 合金、金属氧化物、金属硫化物/磷化物、金属单 原子等)以及面向高电流密度的电极应用(气体扩 散电极)等角度, 对一体化E- $\mathrm{CO}_{2} \mathrm{RR}$ 催化电极进行 了系统综述(图8, 表1)。除此之外,一体化电极在 一系列电化学反应, 如氧还原反应(ORR) ${ }^{121-124}$ 、 析氧反应(OER) ${ }^{125-127}$ 、HER 118,119,128,129、有机小 分子氧化 ${ }^{90,130,131}$ 等中均表现出了优异的催化性能 和潜在的实际应用价值。

尽管一体化电极在 E- $\mathrm{CO}_{2} \mathrm{RR}$ 方面具有重要潜 力, 但在基础研究和应用基础研究方面仍面临着 机遇与挑战。

\section{1 高效一体化电极合理设计与制备}

通过对催化剂的形貌和表界面结构进行精细 调控, 可以影响催化剂表面 $\mathrm{CO}_{2}$ 浓度、催化剂-电解 液界面的 $\mathrm{pH}$ 、反应物和产物传质过程以及催化剂 表面亲疏水性等, 进而有望改善催化电极 E$\mathrm{CO}_{2} \mathrm{RR}$ 的活性、选择性和稳定性等。目前广泛使用 的制备方法主要包括水/溶剂热法、电化学沉积法、 气相沉积法和模板法等。上述方法在电极有序构 筑、活性位利用、制备成本等方面还需进一步优 化。一体化电极如要实现商业化的应用, 必然要开 发简便、高效、低成本的合成策略。在各种合成策

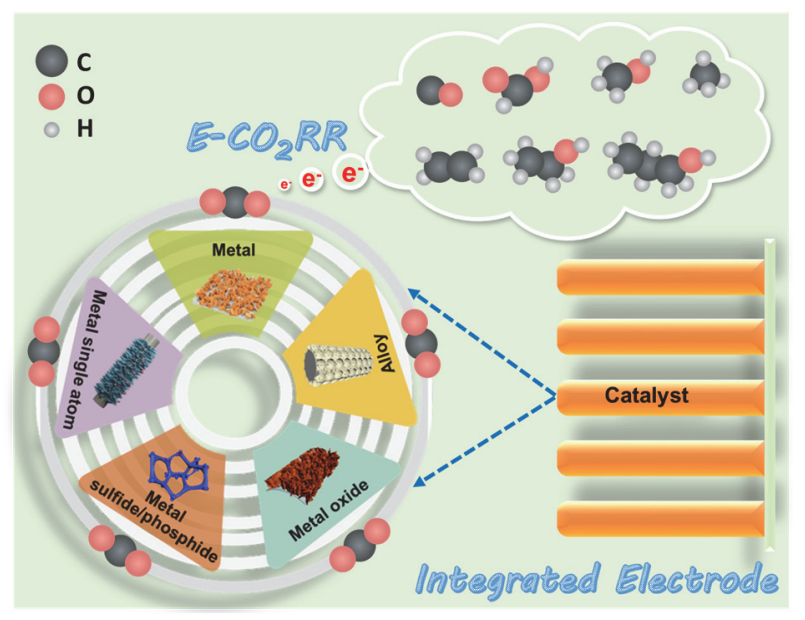

图 8 不同种类的一体化电极用于 $\mathrm{E}-\mathrm{CO}_{2} \mathrm{RR}{ }^{40,47,78,99,110}$

Fig. 8 Different types of integrated electrode for E-CO2 $\operatorname{RR}^{40,47,78,99,110}$.

Adapted from Elsevier ${ }^{40,47}$, Royal Society of Chemistry ${ }^{78,99}$ and Wiley ${ }^{110}$ publications. 
表 1 一体化电极 E-COO2RR 性能汇总

Table 1 Summary of integrated electrode for $\mathrm{E}-\mathrm{CO}_{2} \mathrm{RR}$.

\begin{tabular}{|c|c|c|c|c|c|c|c|}
\hline Catalyst & Product & Electrolyte & Potential/(V vs. RHE) & $\mathrm{FE} / \%$ & $j /\left(\mathrm{mA} \cdot \mathrm{cm}^{-2}\right)$ & Stability/h & Ref. \\
\hline $\mathrm{Zn}$ & $\mathrm{CO}$ & $1.0 \mathrm{~mol} \cdot \mathrm{L}^{-1} \mathrm{KOH}$ & -0.62 & 91.6 & -200 (flow cell) & 18 & 40 \\
\hline Hierarchical $\mathrm{CoS}_{2}$ nanocages & $\mathrm{CO}$ & $0.5 \mathrm{~mol} \cdot \mathrm{L}^{-1} \mathrm{KHCO}_{3}$ & -0.6 & 85.7 & -3.3 & - & 46 \\
\hline $\mathrm{Au}_{3} \mathrm{Cu}$ & $\mathrm{CO}$ & $0.1 \mathrm{~mol} \cdot \mathrm{L}^{-1} \mathrm{KHCO}_{3}$ & -0.7 & 98.12 & -12.77 & 80 & 72 \\
\hline NiSA/PCFM & $\mathrm{CO}$ & $0.5 \mathrm{~mol} \cdot \mathrm{L}^{-1} \mathrm{KHCO}_{3}$ & -1.0 & 88 & -350.4 (flow cell) & 120 & 108 \\
\hline Ag-deposited Ti GDE & $\mathrm{CO}$ & $1.0 \mathrm{~mol} \cdot \mathrm{L}^{-1} \mathrm{KHCO}_{3}$ & - & 67.3 & - & - & 111 \\
\hline NSHCF900 & $\mathrm{CO}$ & $0.5 \mathrm{~mol} \cdot \mathrm{L}^{-1} \mathrm{KHCO}_{3}$ & -0.7 & 94 & -109.5 & 36 & 112 \\
\hline Bi nanodendrite & $\mathrm{HCOO}^{-} / \mathrm{HCOOH}$ & $0.5 \mathrm{~mol} \cdot \mathrm{L}^{-1} \mathrm{NaHCO}_{3}$ & -1.8 (vs. SCE) & 96.4 & -15.2 & 10 & 42 \\
\hline $\mathrm{mPd} / \mathrm{TNTAs}$ & $\mathrm{HCOO}^{-} / \mathrm{HCOOH}$ & $0.5 \mathrm{~mol} \cdot \mathrm{L}^{-1} \mathrm{NaHCO}_{3}$ & -0.1 & $88 \pm 2$ & - & 4 & 57 \\
\hline Binanoflaks@Cu film & $\mathrm{HCOO}^{-} / \mathrm{HCOOH}$ & $0.1 \mathrm{~mol} \cdot \mathrm{L}^{-1} \mathrm{KHCO}_{3}$ & -0.6 & 100 & - & 10 & 62 \\
\hline $\mathrm{V}_{\mathrm{O}}-\mathrm{SnO}_{x} / \mathrm{CF}-40$ & $\mathrm{HCOO}^{-} / \mathrm{HCOOH}$ & $0.1 \mathrm{~mol} \cdot \mathrm{L}^{-1} \mathrm{KHCO}_{3}$ & -1.0 & 86 & -34.9 & 8 & 78 \\
\hline NiSn-APC & $\mathrm{HCOO}^{-} / \mathrm{HCOOH}$ & $0.5 \mathrm{~mol} \cdot \mathrm{L}^{-1} \mathrm{KHCO}_{3}$ & -0.82 & 86.1 & -43.7 & 23 & 110 \\
\hline CuS-NW@NF & $\mathrm{CH}_{4}$ & $0.1 \mathrm{~mol} \cdot \mathrm{L}^{-1} \mathrm{KHCO}_{3}$ & -1.1 & $73 \pm 5$ & - & 60 & 99 \\
\hline Oxide-derived $\mathrm{Cu}$ & $\mathrm{CH}_{4}$ & $0.1 \mathrm{~mol} \cdot \mathrm{L}^{-1} \mathrm{CsHCO}_{3}$ & -1.2 & 62 & -29 & - & 113 \\
\hline Plasma-Cu & $\mathrm{C}_{2} \mathrm{H}_{4}$ & $0.1 \mathrm{~mol} \cdot \mathrm{L}^{-1} \mathrm{NaHCO}_{3}$ & -0.9 & 60 & - & 1 & 114 \\
\hline $\mathrm{Cu}_{2} \mathrm{O}$ films & $\mathrm{C}_{2} \mathrm{H}_{4}$ & $0.1 \mathrm{~mol} \cdot \mathrm{L}^{-1} \mathrm{KHCO}_{3}$ & -0.99 & 39 & -11.7 & - & 115 \\
\hline Porous copper foil & $\mathrm{C}_{2} \mathrm{H}_{4}$ & $0.1 \mathrm{~mol} \cdot \mathrm{L}^{-1} \mathrm{KHCO}_{3}$ & -1.3 & 35 & -57 & 1.4 & 116 \\
\hline FeP NA/TM & $\mathrm{CH}_{3} \mathrm{OH}$ & $0.5 \mathrm{~mol} \cdot \mathrm{L}^{-1} \mathrm{KHCO}_{3}$ & -0.2 & 80.2 & - & 36 & 100 \\
\hline CuSAs/TCNFs & $\mathrm{CH}_{3} \mathrm{OH}$ & $0.1 \mathrm{~mol} \cdot \mathrm{L}^{-1} \mathrm{KHCO}_{3}$ & -0.9 & 44 & -90 & 50 & 117 \\
\hline Zn dendrites & $\mathrm{CH}_{3} \mathrm{OH}$ & $0.5 \mathrm{~mol} \cdot \mathrm{L}^{-1} \mathrm{KHCO}_{3}$ & -0.2 & 10.5 & -26 & - & 118 \\
\hline $\mathrm{AgCu}$ wire & $\mathrm{C}_{2} \mathrm{H}_{5} \mathrm{OH}$ & $1.0 \mathrm{~mol} \cdot \mathrm{L}^{-1} \mathrm{KOH}$ & -0.68 & 30 & -267 (flow cell) & - & 22 \\
\hline $\mathrm{HMMP} \mathrm{Cu}_{5} \mathrm{Zn}_{8}$ & $\mathrm{C}_{2} \mathrm{H}_{5} \mathrm{OH}$ & $0.1 \mathrm{~mol} \cdot \mathrm{L}^{-1} \mathrm{KHCO}_{3}$ & -0.8 & 46.6 & -3.6 & 11 & 47 \\
\hline $\mathrm{Cu}$ nanocrystals & $n$-propanol & $0.1 \mathrm{~mol} \cdot \mathrm{L}^{-1} \mathrm{NaHCO}_{3}$ & -0.95 & 14 & -12 & 6 & 119 \\
\hline $\mathrm{Cu}$ nanowire arrays & $n$-propanol & $0.1 \mathrm{~mol} \cdot \mathrm{L}^{-1} \mathrm{KHCO}_{3}$ & -1.1 & 75 & - & 5 & 120 \\
\hline
\end{tabular}

略中, 如何对催化电极的形貌、组成和结构进行更 加精细地调控还需要进一步探索。如何在制备过 程对所构筑电极的亲疏水性进行可控调控, 进而 研究其与催化性能之间的构效关系仍需进一步探 索; 如何在生长过程中, 加强活性材料与导电基底 之间的相互作用, 以及研究其对电子传递和稳定 性的影响机制还需进一步加强; 如何通过控制反 应条件, 实现高负载量一体化电极的制备, 以满足 实际应用需求，还面临巨大挑战。

\section{2 一体化电极界面揭示与反应强化}

在实际的 $\mathrm{E}-\mathrm{CO}_{2} \mathrm{RR}$ 过程中催化电极通常会发 生表面重构、形貌转变、活性位点的演变/湮灭等 ${ }^{33}$, 如何采用原位技术(原位X射线衍射 (in situ XRD)、 原位红外(in situ IR)、原位拉曼 (in situ Raman)和原 位X射线吸收光谱(in situ XAS)等) ${ }^{132}$, 确定催化活 性位点以及对催化电极的动态演变过程和中间产 物进行实时监测, 深入剖析电极表界面微观结构 变化对催化性能的影响是十分必要的。此外, 电解 液中的碳酸氢根离子是否参与 $\mathrm{E}-\mathrm{CO}_{2} \mathrm{RR}$, 阳离子
对 $\mathrm{E}-\mathrm{CO}_{2} \mathrm{RR}$ 的影响机理以及副反应 HER 与 $\mathrm{E}-$ $\mathrm{CO}_{2} \mathrm{RR}$ 之间的关系尚不明确。目前, E- $\mathrm{CO}_{2} \mathrm{RR}$ 的理 论计算主要依靠热力学数据, 如起始电位、中间体 吸附、吉布斯自由能等。现有的实验结果表明, 电 极的形貌、局部 $\mathrm{pH}$ 值以及电极-电解液界面的离子 种类/浓度对中间体和产物的分布均有影响。然而, 上述影响因素在理论计算时很大程度上被忽视 了。可用于研究不同电势下反应电流与活性组分 的类型/覆盖度之间关系的动力学模型也需被考虑 其中, 这将为一体化 $\mathrm{E}-\mathrm{CO}_{2} \mathrm{RR}$ 电极的精细设计提 供更有利的指导 ${ }^{33}$ 。

\section{3 从一体化电极到新型反应器}

虽然 $\mathrm{H}$ 型电解池被广泛应用于 $\mathrm{E}-\mathrm{CO}_{2} \mathrm{RR}$ 机理 的研究, 但其电流密度受到水溶液中 $\mathrm{CO}_{2}$ 溶解度较 低和扩散速度较慢的限制。GDE辅助的液流电池 能够有效地将 $\mathrm{CO}_{2}$ 输送到电极表面, 从而满足工业 化的电流密度要求 $\left(>300 \mathrm{~mA} \cdot \mathrm{cm}^{-2}\right)$ 。但实现稳定 运行 $(>1000 \mathrm{~h})$, 还需要解决GDE的水淹和堵塞问 题 ${ }^{123}$ 。近年来膜电极组装的电解池也越来越多的 
应用于 $\mathrm{E}-\mathrm{CO}_{2} \mathrm{RR}$ 研究。与液流电池不同, 膜电极组 装的电解池不需要使用液体电解质, 阴阳两极之 间聚合物电解质膜用于离子交换。催化剂直接附 着在膜的两侧或者附着在气体扩散层上置于膜的 两侧 ${ }^{133-136}$ 。受金属-空气电池的启发, 金属- $\mathrm{CO}_{2}$ 电 池(如Li- $\mathrm{CO}_{2}{ }^{137,138} 、 \mathrm{~K}-\mathrm{CO}_{2}{ }^{139}$ 和 Zn- $\mathrm{CO}_{2}$ 电池 ${ }^{140-143}$ 等)近年来也引起了研究者们的关注。但现阶段其 功率密度大小与理论值还存在一定的差距, 电池 的安全稳定性也有待进一步的提升。此外, 在液流 电池或膜电极组装的电解池中, 电池的配置、气体 扩散层、膜、电解质、压力和温度等诸多参数对电 解池的整体性能都有很大的影响 $64,144,145$ 。目前, 和 催化剂电极的设计相比, 这些因素没有得到充分 的重视。一体化电极的不断发展将为未来 $\mathrm{E}-$ $\mathrm{CO}_{2} \mathrm{RR}$ 技术的广泛应用提供更多的可能。

\section{References}

(1) Reichstein, M.; Bahn, M.; Ciais, P.; Frank, D.; Mahecha, M. D.; Seneviratne, S. I.; Zscheischler, J.; Beer, C.; Buchmann, N.; Frank, D. C.; et al. Nature 2013, 500, 287. doi: 10.1038/nature12350

(2) Rogelj, J.; Luderer, G.; Pietzcker, R. C.; Kriegler, E.; Schaeffer, M.; Krey, V.; Riahi, K. Nat. Clim. Change 2015, 5, 519. doi: $10.1038 /$ nclimate 2572

(3) Mac Dowell, N.; Fennell, P. S.; Shah, N.; Maitland, G. C. Nat. Clim. Change 2017, 7, 243. doi: 10.1038/nclimate 3231

(4) Zhu, D. D.; Liu, J. L.; Qiao, S. Z. Adv. Mater. 2016, $28,3423$. doi: 10.1002/adma.201504766

(5) Sreekanth, N.; Nazrulla, M. A.; Vineesh, T. V.; Sailaja, K.; Phani, K. L. Chem. Commun. 2015, 51, 16061. doi: 10.1039/C5CC06051F

(6) Liu, J.; Guo, C.; Vasileff, A.; Qiao, S. Small Methods 2017, 1, 1600006. doi: 10.1002/smtd.201600006

(7) Wu, J. H.; Huang, Y.; Ye, W.; Li, Y. G. Adv. Sci. 2017, 4, 1700194. doi: $10.1002 / \mathrm{advs} .201700194$

(8) Appel, A. M.; Bercaw, J. E.; Bocarsly, A. B.; Dobbek, H.; DuBois, D L.; Dupuis, M.; Ferry, J. G.; Fujita, E.; Hille, R.; Kenis, P. J. A.; et al. Chem. Rev. 2013, 113, 6621. doi: 10.1021/cr300463y

(9) Kumar, B.; Llorente, M.; Froehlich, J.; Dang, T.; Sathrum, A.; Kubiak, C. P. Annu. Rev. Phy. Chem. 2012, 63, 541 doi: 10.1146/annurev-physchem-032511-143759

(10) Furler, P.; Scheffe, J.; Gorbar, M.; Moes, L.; Vogt, U.; Steinfeld, A. Energy Fuels 2012, 26, 7051. doi: 10.1021/ef3013757

(11) Nielsen, D. U.; Hu, X. M.; Daasbjerg, K.; Skrydstrup, T. Nat. Catal. 2018, 1 , 244. doi: 10.1038/s41929-018-0051-3

(12) Wang, W.; Wang, S.; Ma, X.; Gong, J. Chem. Soc. Rev. 2011, 40, 3703. doi: 10.1039/C1CS15008A

(13) Li, F.; Zhao, S. F.; Chen, L.; Khan, A.; MacFarlane, D. R.; Zhang, J.
Energy Environ. Sci. 2016, 9, 216. doi: 10.1039/C5EE02879E

(14) Hong, X.; Chan, K.; Tsai, C.; Nørskov, J. K. ACS Catal. 2016, 6, 4428. doi: 10.1021/acscatal.6b00619

(15) Jin, H.; Guo, C.; Liu, X.; Liu, J.; Vasileff, A.; Jiao, Y.; Zheng, Y.; Qiao, S. Z. Chem. Rev. 2018, 118, 6337. doi: 10.1021/acs.chemrev.7b00689

(16) Wang, Y.; Han, P.; Lv, X.; Zhang, L.; Zheng, G. Joule 2018, 2, 2551. doi: 10.1016/j.joule.2018.09.021

(17) Wang, W.; Shang, L.; Chang, G.; Yan, C.; Shi, R.; Zhao, Y.; Waterhouse, G. I. N.; Yang, D.; Zhang, T. Adv. Mater. 2019, 31, 1808276. doi: 10.1002/adma.201808276

(18) Liu, S.; Yang, H.; Su, X.; Ding, J.; Mao, Q.; Huang, Y.; Zhang, T.; Liu, B. J. Energy Chem. 2019, 36, 95. doi: 10.1016/j.jechem.2019.06.013

(19) Zhou, Y.; Che, F.; Liu, M.; Zou, C.; Liang, Z.; De Luna, P.; Yuan, H.; Li, J.; Wang, Z.; Xie, H.; et al. Nat. Chem. 2018, 10, 974. doi: 10.1038/s41557-018-0092-x

(20) Pan, F.; Li, B.; Sarnello, E.; Hwang, S.; Gang, Y.; Feng, X.; Xiang, X.; Adli, N. M.; Li, T.; Su, D.; et al. Nano Energy 2020, 68, 104384. doi: 10.1016/j.nanoen.2019.104384

(21) Pan, F.; Duan, Y.; Liang, A.; Zhang, J.; Li, Y. Electrochim. Acta 2017, 238, 375. doi: 10.1016/j.electacta.2017.04.044

(22) Hoang, T. T. H.; Verma, S.; Ma, S.; Fister, T. T.; Timoshenko, J.; Frenkel, A. I.; Kenis, P. J. A.; Gewirth, A. A. J. Am. Chem. Soc. 2018, 140, 5791. doi: 10.1021/jacs.8b01868

(23) Wen, G.; Lee, D. U.; Ren, B.; Hassan, F. M.; Jiang, G.; Cano, Z. P.; Gostick, J.; Croiset, E.; Bai, Z.; Yang, L.; et al. Adv. Energy Mater. 2018, 8, 1802427. doi: 10.1002/aenm.201802427

(24) Cheng, T.; Wang, L.; Merinov, B. V.; Goddard, W. A. J. Am. Chem. Soc. 2018, 140, 7787. doi: 10.1021/jacs.8b04006

(25) Li, L.; Ma, D. K.; Qi, F.; Chen, W.; Huang, S. Electrochim. Acta 2019, 298, 580. doi: 10.1016/j.electacta.2018.12.116

(26) Liu, S.; Xiao, J.; Lu, X. F.; Wang, J.; Wang, X.; Lou, X. W. D. Angew. Chem. Int. Ed. 2019, 58, 8499. doi: 10.1002/anie.201903613

(27) Liang, C.; Kim, B.; Yang, S.; Yang, L.; Francisco Woellner, C.; Li, Z.; Vajtai, R.; Yang, W.; Wu, J.; Kenis, P. J. A.; et al. J. Mater. Chem. A 2018, 6, 10313. doi: 10.1039/C8TA01367E

(28) Zheng, X.; Ji, Y.; Tang, J.; Wang, J.; Liu, B.; Steinrück, H. G.; Lim, K.; Li, Y.; Toney, M. F.; Chan, K.; et al. Nat. Catal. 2019, $2,55$. doi: $10.1038 / \mathrm{s} 41929-018-0200-8$

(29) Nitopi, S.; Bertheussen, E.; Scott, S. B.; Liu, X.; Engstfeld, A. K.; Horch, S.; Seger, B.; Stephens, I. E. L.; Chan, K.; Hahn, C.; et al. Chem. Rev. 2019, 119, 7610. doi: 10.1021/acs.chemrev.8b00705

(30) Zhao, Y.; Tan, X.; Yang, W.; Jia, C.; Chen, X.; Ren, W.; Smith, S. C.; Zhao, C. Angew. Chem. Int. Ed. 2020, 59, 21493. doi: 10.1002/anie.202009616 
(31) Michael B. Ross; Yang, P.; Phil De Luna; Yi, F. L.; Cao Thang Dinh; Dohyung Kim; Sargent, E. H. Nat. Catal. 2019, 2, 648. doi: 10.1038/s41929-019-0306-7

(32) Liu, X.; Xiao, J.; Peng, H.; Hong, X.; Chan, K.; Nørskov, J. K. Nat. Commun. 2017, 8, 15438. doi: 10.1038/ncomms 15438

(33) Pan, F.; Yang, Y. Energy Environ. Sci. 2020, 13, 2275. doi: $10.1039 / \mathrm{d} 0 \mathrm{ee} 00900 \mathrm{~h}$

(34) Sun, H.; Yan, Z.; Liu, F.; Xu, W.; Cheng, F.; Chen, J. Adv. Mater. 2020, 32, 1806326. doi: 10.1002/adma.201806326

(35) Liu, J.; Zhu, D.; Zheng, Y.; Vasileff, A.; Qiao, S. ACS Catal. 2018, 8, 6707. doi: 10.1021/acscatal.8b01715

(36) Yang, H.; Wang, X.; Hu, Q.; Chai, X.; Ren, X.; Zhang, Q.; Liu, J.; He, C. Small Methods 2020, 4, 1900826. doi: 10.1002/smtd.201900826

(37) Tang, C.; Wang, H. F.; Zhang, Q. Acc. Chem. Res. 2018, 51, 881. doi: 10.1021/acs.accounts. $7 \mathrm{~b} 00616$

(38) Ji, D. X.; Fan, L.; Li, L. L.; Peng, S. J.; Yu, D. S.; Song, J. N.; Ramakrishna, S.; Guo, S. J. Adv. Mater. 2019, 31, 1808267. doi: 10.1002/adma.201808267

(39) Wang, P.; Jia, T.; Wang, B. J. Power Sources 2020, 474, 228621. doi: 10.1016/j.jpowsour.2020.228621

(40) Luo, W.; Zhang, Q.; Zhang, J.; Moioli, E.; Zhao, K.; Züttel, A. Appl. Catal. B: Environ. 2020, 273, 119060. doi: 10.1016/j.apcatb.2020.119060

(41) Zhang, T.; Han, X.; Yang, H.; Han, A.; Hu, E.; Li, Y.; Yang, X. Q.; Wang, L.; Liu, J.; Liu, B. Angew. Chem. Int. Ed. 2020, 59, 2. doi: 10.1002/anie.202002984

(42) Zhong, H.; Qiu, Y.; Zhang, T.; Li, X.; Zhang, H.; Chen, X. J. Mater. Chem. A 2016, 4, 13746. doi: 10.1039/c6ta06202d

(43) Zhou, L.; Shao, M. F.; Li, J. B.; Jiang, S.; Wei, M.; Duan, X. Nano Energy 2017, 41, 583. doi: 10.1016/j.nanoen.2017.10.009

(44) Xu, K.; Wang, F.; Wang, Z.; Zhan, X.; Wang, Q.; Cheng, Z.; Safdar, M.; He, J. ACS Nano 2014, 8, 8468. doi: 10.1021/nn503027k

(45) Li, Z. H.; Shao, M. F.; Yang, Q. H.; Tang, Y.; Wei, M.; Evans, D. G.; Duan, X. Nano Energy 2017, 37, 98. doi: 10.1016/j.nanoen.2017.05.016

(46) Han, Z.; Hu, Q.; Cheng, Z.; Li, G.; Huang, X.; Wang, Z.; Yang, H.; Ren, X.; Zhang, Q.; Liu, J.; et al. Adv. Funct. Mater. 2020, 30, 2000154. doi: 10.1002/adfm.202000154

(47) Su, X.; Sun, Y.; Jin, L.; Zhang, L.; Yang, Y.; Kerns, P.; Liu, B.; Li, S.; He, J. Appl. Catal. B: Environ. 2020, 269, 118800. doi: 10.1016/j.apcatb.2020.118800

(48) An, X.; Li, S.; Yoshida, A.; Yu, T.; Wang, Z.; Hao, X.; Abudula, A.; Guan, G. ACS Appl. Mater. Interfaces 2019, 11, 42114. doi: 10.1021 /acsami.9b13270

(49) Rosen, J.; Hutchings, G. S.; Lu, Q.; Forest, R. V.; Moore, A.; Jiao, F. ACS Catal. 2015, 5, 4586. doi: 10.1021/acscatal.5b00922

(50) Wanninayake, N.; Ai, Q.; Zhou, R.; Hoque, M. A.; Herrell, S.;
Guzman, M. I.; Risko, C.; Kim, D. Y. Carbon 2020, 157, 408. doi: 10.1016/j.carbon.2019.10.022

(51) Hori, Y.; Kikuchi, K.; Murata, A.; Suzuki, S. Chem. Lett. 1986, 15, 897. doi: $10.1246 / \mathrm{cl} .1986 .897$

(52) Hori, Y.; Wakebe, H.; Tsukamoto, T.; Koga, O. Electrochim. Acta 1994, 39, 1833. doi: 10.1016/0013-4686(94)85172-7

(53) Hori, Y.; Murata, A.; Takahashi, R. J. Chem. Soc., Faraday Trans. 1 1989, 85, 2309. doi: 10.1039/F19898502309

(54) Kuhl, K. P.; Cave, E. R.; Abram, D. N.; Jaramillo, T. F. Energy Environ. Sci. 2012, 5, 7050. doi: 10.1039/C2EE21234J

(55) Bai, X. F.; Wei, C.; Wang, B. Y.; Feng, G. H.; WeI, W.; Jiao, Z.; Sun, Y. H. Acta Phys. -Chim. Sin. 2017, 33, 2388. [白晓芳, 陈为, 王白 银, 冯光辉, 魏伟, 焦正, 孙予罕. 物理化学学报, 2017, 33, 2388.] doi: 10.3866/PKU.WHXB201706131

(56) Zhou, Y.; Han, N.; Li, Y. G. Acta Phys. -Chim. Sin. 2020, 36, 2001041. [周远, 韩娜, 李彦光. 物理化学学报, 2020, 36, 2001041.] doi: 10.3866/PKU.WHXB202001041

(57) Zou, J.; Iqbal, M.; Vijayakumar, A.; Wang, C.; Macfarlane, D. R.; Yamauchi, Y.; Lee, C. Y.; Wallace, G. G. J. Mater. Chem. A 2020, 8, 8041. doi: 10.1039/d0ta02077j

(58) Greeley, J.; Jaramillo, T. F.; Bonde, J.; Chorkendorff, I.; Nørskov, J. K. Nat. Mater. 2006, 5, 909. doi: 10.1038/nmat1752

(59) Yang, H.; Han, N.; Deng, J.; Wu, J.; Wang, Y.; Hu, Y.; Ding, P.; Li, Y.; Li, Y.; Lu, J. Adv. Energy Mater. 2018, 8, 1801536. doi: 10.1002/aenm.201801536

(60) Gong, Q.; Ding, P.; Xu, M.; Zhu, X.; Wang, M.; Deng, J.; Ma, Q.; Han, N.; Zhu, Y.; Lu, J.; et al. Nat. Commun. 2019, 10, 2807. doi: 10.1038/s41467-019-10819-4

(61) Han, N.; Wang, Y.; Yang, H.; Deng, J.; Wu, J. H.; Li, Y. F.; Li, Y. G. Nat. Commun. 2018, 9, 1320. doi: 10.1038/s41467-018-03712-z

(62) Kim, S.; Dong, W. J.; Gim, S.; Sohn, W.; Park, J. Y.; Yoo, C. J.; Jang, H. W.; Lee, J. L. Nano Energy 2017, 39, 44. doi: 10.1016/j.nanoen.2017.05.065

(63) Cai, Z.; Zhang, Y.; Zhao, Y.; Wu, Y.; Xu, W.; Wen, X.; Zhong, Y.; Zhang, Y.; Liu, W.; Wang, H.; et al. Nano Res. 2018, 12, 345. doi: $10.1007 / \mathrm{s} 12274-018-2221-7$

(64) Kibria, M. G.; Edwards, J. P.; Gabardo, C. M.; Dinh, C. T.; Seifitokaldani, A.; Sinton, D.; Sargent, E. H. Adv. Mater. 2019, 31, 1807166. doi: 10.1002/adma.201807166

(65) Zhuang, T. T.; Liang, Z. Q.; Seifitokaldani, A.; Li, Y.; De Luna, P.; Burdyny, T.; Che, F.; Meng, F.; Min, Y.; Quintero Bermudez, R.; et al. Nat. Catal. 2018, 1, 421. doi: 10.1038/s41929-018-0084-7

(66) Dinh, C. T.; Burdyny, T.; Kibria, M. G.; Seifitokaldani, A.; Gabardo, C. M.; García de Arquer, F. P.; Kiani, A.; Edwards, J. P.; De Luna, P.; Bushuyev, O. S.; et al. Science 2018, 360, 783. doi: $10.1126 /$ science.aas 9100 
(67) Burdyny, T.; Smith, W. A. Energy Environ. Sci. 2019, 12, 1431. doi: 10.1039/C8EE03134G

(68) Weekes, D. M.; Salvatore, D. A.; Reyes, A.; Huang, A.; Berlinguette, C. P. Acc. Chem. Res. 2018, 51, 910 . doi: 10.1021/acs.accounts. 8 b00010

(69) Sedighian Rasouli, A.; Wang, X.; Wicks, J.; Lee, G.; Peng, T.; Li, F.; McCallum, C.; Dinh, C. T.; Ip, A. H.; Sinton, D.; et al. ACS Sustainable Chem. Eng. 2020, 8, 14668. doi: $10.1021 /$ acssuschemeng.0c03453

(70) Li, Y.; Cui, F.; Ross, M. B.; Kim, D.; Sun, Y.; Yang, P. Nano Lett. 2017, 17, 1312. doi: 10.1021/acs.nanolett.6b05287

(71) Shao, J.; Wang, Y.; Gao, D.; Ye, K.; Wang, Q.; Wang, G. Chin. J. Catal. 2020, 41, 1393. doi: 10.1016/s1872-2067(20)63577-x

(72) Ma, X.; Shen, Y.; Yao, S.; Shu, M.; Si, R.; An, C. Chem. Eur. J. 2019, 26, 4143. doi: 10.1002/chem.201904619

(73) Li, Y. C.; Wang, Z.; Yuan, T.; Nam, D. H.; Luo, M.; Wicks, J.; Chen, B.; Li, J.; Li, F.; de Arquer, F. P. G.; et al. J. Am. Chem. Soc. 2019, 141, 8584. doi: $10.1021 /$ jacs.9b02945

(74) Schreier, M.; Héroguel, F.; Steier, L.; Ahmad, S.; Luterbacher, J. S.; Mayer, M. T.; Luo, J.; Grätzel, M. Nat. Energy 2017, 2, 17087. doi: $10.1038 /$ nenergy.2017.87

(75) Zhang, E. H.; Wang, T.; Yu, K.; Liu, J.; Chen, W. X.; Li, A.; Rong, H. P.; Lin, R.; Ji, S. F.; Zheng, X. S.; et al. J. Am. Chem. Soc. 2019, 141, 16569. doi: $10.1021 /$ jacs. 9 b08259

(76) Sun, J.; Zheng, W.; Lyu, S.; He, F.; Yang, B.; Li, Z.; Lei, L.; Hou, Y. Chin. Chem. Lett. 2020, 31, 1415. doi: 10.1016/j.cclet.2020.04.031

(77) Hao, L.; Sun, Z. Acta Phys. -Chim. Sin. 2021, 37, 2009033. [郝否端, 孙振宇. 物理化学学报, 2021, 37, 2009033.] doi: 10.3866/PKU.WHXB202009033

(78) Li, H.; Xiao, N.; Wang, Y.; Liu, C.; Zhang, S.; Zhang, H.; Bai, J.; Xiao, J.; Li, C.; Guo, Z.; et al. J. Mater. Chem. A 2020, 8, 1779. doi: $10.1039 / \mathrm{c} 9 \operatorname{ta} 12401 \mathrm{~b}$

(79) Tran Phu, T.; Daiyan, R.; Fusco, Z.; Ma, Z.; Amal, R.; Tricoli, A. Adv. Funct. Mater. 2019, 30, 1906478. doi: 10.1002/adfm.201906478

(80) Wu, D.; Huo, G.; Chen, W.; Fu, X. Z.; Luo, J. L. Appl. Catal. B: Environ. 2020, 271, 118957. doi: 10.1016/j.apcatb.2020.118957

(81) Jouny, M.; Luc, W.; Jiao, F. Ind. Eng. Chem. Res. 2018, 57, 2165. doi: 10.1021/acs.iecr.7b03514

(82) Wang, X. X.; Klingan, K.; Klingenhof, M.; Möller, T.; Araújo, J. F.; Martens, I.; Bagger, A.; Jiang, S.; Rossmeisl, J.; Dau, Holger.; et al. Nat. Commun. 2021, 12, 794. doi: 10.1038/s41467-021-20961-7

(83) Luo, M.; Wang, Z.; Li, Y. C.; Li, J.; Li, F.; Lum, Y.; Nam, D. H.; Chen, B.; Wicks, J.; Xu, A.; et al. Nat. Commun. 2019, 10, 5814. doi: 10.1038/s41467-019-13833-8

(84) Li, J.; Xu, A.; Li, F.; Wang, Z.; Zou, C.; Gabardo, C. M.; Wang, Y.; Ozden, A.; Xu, Y.; Nam, D. H.; et al. Nat. Commun. 2020, 11, 3685. doi: 10.1038/s41467-020-17499-5

(85) Cheng, T.; Xiao, H.; Goddard, W. A. J. Phys. Chem. Lett. 2015, 6, 4767. doi: 10.1021/acs.jpclett.5b02247

(86) Cheng, T.; Xiao, H.; Goddard, W. A. Proc. Natl Acad. Sci. USA 2017, 114, 1795. doi: 10.1073/pnas.1612106114

(87) Li, Y.; Xu, A.; Lum, Y.; Wang, X.; Hung, S. F.; Chen, B.; Wang, Z.; Xu, Y.; Li, F.; Abed, J.; et al. Nat. Commun. 2020, 11, 6190. doi: 10.1038/s41467-020-20004-7

(88) Xie, J.; Zhang, H.; Li, S.; Wang, R.; Sun, X.; Zhou, M.; Zhou, J.; Lou, X. W.; Xie, Y. Adv. Mater. 2013, 25, 5807. doi: 10.1002/adma.201302685

(89) Liu, Y.; Jiang, S.; Li, S.; Zhou, L.; Li, Z.; Li, J.; Shao, M. Appl. Catal. B: Environ. 2019, 247, 107. doi: 10.1016/j.apcatb.2019.01.094

(90) Zhou, L.; Shao, M. F.; Zhang, C.; Zhao, J.; He, S.; Rao, D.; Wei, M.; Evans, D. G.; Duan, X. Adv. Mater. 2017, 29, 1604080. doi: 10.1002/adma.201604080

(91) Deng, X.; Kang, X.; Li, M.; Xiang, K.; Wang, C.; Guo, Z.; Zhang, J.; Fu, X. Z.; Luo, J. L. J. Mater. Chem. A 2020, 8, 1138. doi: $10.1039 / \mathrm{c} 9 \mathrm{ta} 06917 \mathrm{~h}$

(92) Gu, J.; Aguiar, J. A.; Ferrere, S.; Steirer, K. X.; Yan, Y.; Xiao, C.; Young, James L.; Al Jassim, M.; Neale, N. R.; Turner, J. A. Nat. Energy 2017, 2, 16192. doi: 10.1038/nenergy.2016.192

(93) Wang, J.; Chao, D.; Liu, J.; Li, L.; Lai, L.; Lin, J.; Shen, Z. Nano Energy 2014, 7, 151. doi: 10.1016/j.nanoen.2014.04.019

(94) Asadi, M.; Kumar, B.; Behranginia, A.; Rosen, B. A.; Baskin, A.; Repnin, N.; Pisasale, D.; Phillips, P.; Zhu, W.; Haasch, R.; et al. Nat. Commun. 2014, 5, 4470. doi: 10.1038/ncomms5470

(95) Zhu, Q.; Sun, X.; Kang, X.; Ma, J.; Qian, Q.; Han, B. Acta Phys. -Chim. Sin. 2016, 32, 261. [ 朱庆宫, 孙哓甫, 康欣晨, 马珺, 钱庆利, 韩布兴. 物理化学学报, 2016, 32, 261.] doi: 10.3866/PKU.WHXB201512101

(96) Xu, J.; Li, X.; Liu, W.; Sun, Y.; Ju, Z.; Yao, T.; Wang, C.; Ju, H.; Zhu, J.; Wei, S.; et al. Angew. Chem. Int. Ed. 2017, 56, 9121. doi: 10.1002/anie. 201704928

(97) Shi, G.; Yu, L.; Ba, X.; Zhang, X.; Zhou, J.; Yu, Y. Dalton Trans. 2017, 46, 10569. doi: 10.1039/C6DT04381J

(98) Kong, X.; Wang, C.; Zheng, H.; Geng, Z.; Bao, J.; Zeng, J. Sci. China Chem. 2021, 64, 1096. doi: 10.1007/s11426-020-9934-0

(99) Zhao, Z.; Peng, X. Y.; Liu, X. J.; Sun, X. M.; Shi, J.; Han, L. 1.; Lia, G. L.; Luo, J. J. Mater. Chem. A 2017, 5, 20239. doi: 10.1039/C7TA05507B

(100) Ji, L.; Li, L.; Ji, X.; Zhang, Y.; Mou, S.; Wu, T.; Liu, Q.; Li, B.; Zhu, X.; Luo, Y.; et al. Angew. Chem. Int. Ed. 2019, 132, 768. doi: 10.1002/anie.201912836

(101) Landers, A. T.; Fields, M.; Torelli, D. A.; Xiao, J.; Hellstern, T. R.; 
Francis, S. A.; Tsai, C.; Kibsgaard, J.; Lewis, N. S.; Chan, K.; et al. ACS Energy Lett. 2018, 3, 1450.

doi: 10.1021/acsenergylett.8b00237

(102) Gu, J.; Hsu, C. S.; Bai, L.; Chen, H. M.; Hu, X. Science 2019, 364, 1091. doi: 10.1126/science.aaw7515

(103) Wang, A.; Li, J.; Zhang, T. Nat. Rev. Chem. 2018, 2, 65. doi: 10.1038/s41570-018-0010-1

(104) Yang, H. B.; Hung, S. F.; Liu, S.; Yuan, K.; Miao, S.; Zhang, L.; Huang, X.; Wang, H. Y.; Cai, W.; Chen, R.; et al. Nat. Energy 2018, 3, 140. doi: 10.1038/s41560-017-0078-8

(105) Pan, F.; Zhang, H.; Liu, Z.; Cullen, D.; Liu, K.; More, K.; Wu, G.; Wang, G.; Li, Y. J. Mater. Chem. A 2019, 7, 26231. doi: 10.1039/C9TA08862H

(106) Cheng, Y.; Zhao, S.; Johannessen, B.; Veder, J. P.; Saunders, M.; Rowles, M. R.; Cheng, M.; Liu, C.; Chisholm, M. F.; De Marco, R.; et al. Adv. Mater. 2018, 30, 1706287. doi: 10.1002/adma.201706287

(107) Huang, X.; Ma, Y.; Zhi, L. Acta Phys. -Chim. Sin. 2021, 37 , 2011050. [黄小雄, 马英杰, 智林杰. 物理化学学报, 2021, 37, 2011050.] doi: 10.3866/PKU.WHXB202011050

(108) Yang, H.; Lin, Q.; Zhang, C.; Yu, X.; Cheng, Z.; Li, G.; Hu, Q.; Ren, X.; Zhang, Q.; Liu, J.; et al. Nat. Commun. 2020, 11, 593. doi: 10.1038/s41467-020-14402-0

(109) Zhao, C.; Wang, Y.; Li, Z.; Chen, W.; Xu, Q.; He, D.; Xi, D.; Zhang, Q.; Yuan, T.; Qu, Y.; et al. Joule 2018, 3, 584. doi: 10.1016/j.joule.2018.11.008

(110) Xie, W.; Li, H.; Cui, G.; Li, J.; Song, Y.; Li, S.; Zhang, X.; Lee, J. Y.; Shao, M.; Wei, M. Angew. Chem. Int. Ed. 2021, 60, 7382. doi: 10.1002/anie.202014655

(111) Oh, S.; Park, Y. S.; Park, H.; Kim, H.; Jang, J. H.; Choi, I.; Kim, S. K. J. Ind. and Eng. Chem. 2020, 82, 374. doi: 10.1016/j.jiec.2019.11.001

(112) Yang, H. P.; Wu, Y.; Lin, Q.; Fan, L. D.; Chai, X. Y.; Zhang, Q. L.; Liu, J. H.; He, C. X.; Lin, Z. Q. Angew. Chem. Int. Ed. 2018, 57, 15476. doi: 10.1002/anie.201809255

(113) Ren, D.; Fong, J.; Yeo, B. S. Nat. Commun. 2018, 9, 925. doi: 10.1038/s41467-018-03286-w

(114) Mistry, H.; Varela, A. S.; Bonifacio, C. S.; Zegkinoglou, I.; Sinev, I.; Choi, Y. W.; Kisslinger, K.; Stach, E. A.; Yang, J. C.; Strasser, P.; et al. Nat. Commun. 2016, 7, 12123. doi: 10.1038/ncomms 12123

(115) Ren, D.; Deng, Y.; Handoko, A. D.; Chen, C. S.; Malkhandi, S.; Yeo, B. S. ACS Catal. 2015, 5, 2814. doi: 10.1021/cs502128q

(116) Peng, Y.; Wu, T.; Sun, L.; Nsanzimana, J. M. V.; Fisher, A. C.; Wang, X. ACS Appl. Mater. Interfaces 2017, 9, 32782. doi: 10.1021/acsami.7b10421

(117) Yang, H.; Wu, Y.; Li, G.; Lin, Q.; Hu, Q.; Zhang, Q.; Liu, J.; He, C. J. Am. Chem. Soc. 2019, 141, 12717. doi: 10.1021/jacs.9b04907
(118) Low, Q. H.; Loo, N. W. X.; Calle Vallejo, F.; Yeo, B. S. Angew. Chem. Int. Ed. 2019, 58, 2256. doi: 10.1002/anie.201810991

(119) Ren, D.; Wong, N. T.; Handoko, A. D.; Huang, Y.; Yeo, B. S. J. Phys. Chem. Lett. 2016, 7, 20. doi: 10.1021/acs.jpclett.5b02554

(120) Ma, M.; Djanashvili, K.; Smith, W. A. Angew. Chem. Int. Ed. 2016, 55, 6680. doi: 10.1002/anie.201601282

(121) Xie, W.; Song, Y.; Li, S.; Li, J.; Yang, Y.; Liu, W.; Shao, M.; Wei, M. Adv. Funct. Mater. 2019, 29, 1906477. doi: $10.1002 /$ adfm. 201906477

(122) Fan, K.; Li, Z.; Song, Y.; Xie, W.; Shao, M.; Wei, M. Adv. Funct. Mater. 2020, 2008064. doi: 10.1002/adfm.202008064

(123) Li, S.; Xie, W.; Song, Y.; Shao, M. ACS Sustainable Chem. Eng. 2020, 8, 452. doi: 10.1021/acssuschemeng.9b05754

(124) Xie, W.; Li, J.; Song, Y.; Li, S.; Li, J.; Shao, M. Nano-Micro Lett. 2020, 12, 97. doi: 10.1007/s40820-020-00435-z

(125) Xiao, K.; Zhou, L.; Shao, M.; Wei, M. J. Mater. Chem. A 2018, 6, 7585. doi: $10.1039 / \mathrm{c} 8 \mathrm{ta} 01067 \mathrm{f}$

(126) Zhou, L.; Jiang, S.; Liu, Y.; Shao, M.; Wei, M.; Duan, X. ACS Appl. Energy Mater. 2018, 1, 623. doi: 10.1021/acsaem.7b00151

(127) Jiang, S.; Liu, Y.; Xie, W.; Shao, M. J. Energy Chem. 2019, 33, 125. doi: 10.1016/j.jechem.2018.08.010

(128) Huang, X.; Xu, X.; Luan, X.; Cheng, D. Nano Energy 2020, 68, 104332. doi: 10.1016/j.nanoen.2019.104332

(129) Yan, L.; Zhang, B.; Zhu, J.; Li, Y.; Tsiakaras, P.; Kang Shen, P. Appl. Catal. B: Environ. 2020, 265, 118555. doi: 10.1016/j.apcatb.2019.118555

(130) Liu, W.; Dang, L.; Xu, Z.; Yu, H.; Jin, S.; Huber, G. W. ACS Catal. 2018, 8, 5533. doi: 10.1021/acscatal.8b01017

(131) Zhang, M.; Liu, Y.; Liu, B.; Chen, Z.; Xu, H.; Yan, K. ACS Catal. 2020, 10, 5179. doi: 10.1021/acscatal.0c00007

(132) Li, X.; Wang, S.; Li, L.; Sun, Y.; Xie, Y. J. Am. Chem. Soc. 2020, 142, 9567. doi: 10.1021/jacs.0c02973

(133) Han, N.; Ding, P.; He, L.; Li, Y. Y.; Li, Y. G. Adv. Energy Mater. 2019, 10, 1902338. doi: 10.1002/aenm.201902338

(134) Lee, W.; Kim, Y. E.; Youn, M. H.; Jeong, S. K.; Park, K. T. Angew. Chem. Int. Ed. 2018, 57, 6883. doi: 10.1002/anie.201803501

(135) Yin, Z.; Peng, H.; Wei, X.; Zhou, H.; Gong, J.; Huai, M.; Xiao, L.; Wang, G.; Lu, J.; Zhuang, L. Energy Environ. Sci. 2019, 12, 2455. doi: $10.1039 / \mathrm{c} 9 \mathrm{ee} 01204 \mathrm{~d}$

(136) Wei, P.; Li, H.; Lin, L.; Gao, D.; Zhang, X.; Gong, H.; Qing, G.; Cai, R.; Wang, G.; Bao, X. Sci. China. Chem. 2020, 63, 1711. doi: 10.1007/s11426-020-9825-9

(137) Hu, C.; Gong, L.; Xiao, Y.; Yuan, Y.; Bedford, N. M.; Xia, Z.; Ma, L.; Wu, T.; Lin, Y.; Connell, J. W.; et al. Adv. Mater. 2020, 1907436. doi: 10.1002/adma.201907436

(138) Chen, J.; Zou, K.; Ding, P.; Deng, J.; Zha, C.; Hu, Y.; Zhao, X.; Wu, 
J.; Fan, J.; Li, Y. Adv. Mater. 2019, 31, 1805484. doi: 10.1002/adma.201805484

(139) Zhang, W.; Hu, C.; Guo, Z.; Dai, L. Angew. Chem. Int. Ed. 2020, 59, 3470. doi: 10.1002/anie. 201913687

(140) Wang, K.; Wu, Y.; Cao, X.; Gu, L.; Hu, J. Adv. Funct. Mater. 2020, 30, 1908965. doi: 10.1002/adfm.201908965

(141) Zheng, W.; Yang, J.; Chen, H.; Hou, Y.; Wang, Q.; Gu, M.; He, F.; Xia, Y.; Xia, Z.; Li, Z.; et al. Adv. Funct. Mater. 2019, 30, 1907658. doi: 10.1002/adfm.201907658

(142) Xie, J.; Wang, X.; Lv, J.; Huang, Y.; Wu, M.; Wang, Y.; Yao, J. Angew. Chem. Int. Ed. 2018, 57, 16996. doi: $10.1002 /$ anie 201811853

(143) Yang, R.; Xie, J.; Liu, Q.; Huang, Y.; Lv, J.; Ghausi, M. A.; Wang, X.; Peng, Z.; Wu, M.; Wang, Y. J. Mater. Chem. A 2019, 7, 2575. doi: $10.1039 / \mathrm{c} 8 \mathrm{ta} 10958 \mathrm{c}$

(144) Endrődi, B.; Bencsik, G.; Darvas, F.; Jones, R.; Rajeshwar, K.; Janáky, C. Prog. Energy Combust. 2017, 62, 133. doi: 10.1016/j.pecs.2017.05.005

(145) Gao, D.; Wei, P.; Li, H.; Lin, L.; Wang, G.; Bao, X. Acta Phys. -Chim. Sin. 2021, 37, 2009021. [高敦峰, 魏鹏飞, 李合肥, 林龙, 汪国雄, 包信和. 物理化学学报, 2021, 37, 2009021.] doi: 10.3866/PKU.WHXB202009021 